Portland State University

PDXScholar

$1-11-2020$

\title{
Characterization of Inertial Particles in the Turbulent Wake of a Porous Disk
}

Kristin Nichole Travis

Portland State University

Follow this and additional works at: https://pdxscholar.library.pdx.edu/open_access_etds

Part of the Aerodynamics and Fluid Mechanics Commons, and the Mechanical Engineering Commons Let us know how access to this document benefits you.

\section{Recommended Citation}

Travis, Kristin Nichole, "Characterization of Inertial Particles in the Turbulent Wake of a Porous Disk" (2020). Dissertations and Theses. Paper 5641.

https://doi.org/10.15760/etd.7513

This Thesis is brought to you for free and open access. It has been accepted for inclusion in Dissertations and Theses by an authorized administrator of PDXScholar. Please contact us if we can make this document more accessible: pdxscholar@pdx.edu. 
Characterization of Inertial Particles in the Turbulent Wake of a Porous Disk

by

Kristin Nichole Travis

A thesis submitted in partial fulfillment of the

requirements for the degree of

\author{
Master of Science \\ in \\ Mechanical Engineering
}

Thesis Committee:

Raúl Bayoán Cal, Chair

Elliott Gall

Gerald Recktenwald

Portland State University

2020 


\begin{abstract}
This study presents the findings of a wind tunnel experiment investigating the behaviour of micrometric inertial particles in the turbulent wake of a stationary porous disk. Various concentrations $\left[\phi_{v} \in\left(2.95 \times 10^{-6}-1.22 \times 10^{-5}\right)\right]$ of polydisperse water droplets (diameter 13-41 $\mu \mathrm{m}$ ) are compared to sub-inertial tracer particles. Hot-wire anemometry, phase Doppler interferometry and particle image velocimetry were implemented in the near and far wake regions to study the complex dynamics of the particles. Turbulence statistics and particle size distributions are presented and used to explore the particle wake interaction.
\end{abstract}




\section{Acknowledgements}

I would like to express my sincerest gratitude to my advisors Raúl Bayoán Cal and Martín Obligado for continuous guidance and support of this research. I would also like to thank members of the research group, including Sarah E. Smith, Bianca Viggiano, Hawwa Kadum, and Naseem Ali for their guidance, expertise and insight from their studies. Finally, the Laboratoire des Écoulements Géophysiques et Industriels (LEGI), Université Grenoble Alpes (Grenoble, France) and specifically Henda Djeridi, and Laure Vignal for their technical support and guidance. 


\section{Contents}

Abstract

$\begin{array}{ll}\text { Acknowledgements ii } & \text { ii }\end{array}$

List of Tables $\quad$ v

List of Figures

Chapter 1 Introduction 1

$\begin{array}{lll}\text { Chapter } 2 & \text { Theory } & 6\end{array}$

2.1 Turbulence Statistics .................... 6

2.2 Multiphase flow .................... 8

$\begin{array}{lll}\text { Chapter } 3 \text { Experimental Setup } & 10\end{array}$

3.1 Inflow Characterization $\ldots \ldots \ldots \ldots \ldots \ldots$

3.2 Two-phase Flow Experiments . . . . . . . . . . . . . . . . . . . . 14

$\begin{array}{llr}\text { Chapter } 4 & \text { Results } & 18\end{array}$

4.1 Hot-wire Anemometry . . . . . . . . . . . . . . . . 18

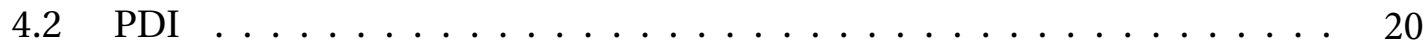

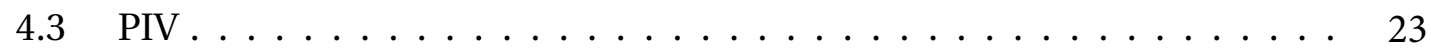


Chapter 5 Discussion

Chapter 6 Conclusion

Chapter 7 Future Work

Bibliography 
List of Tables

3.1 Calculated Turbulence Statistics for the Background Flow . . . . . . . . 11

3.2 PDI and PIV Case Parameters . . . . . . . . . . . . . . . . 16 


\section{List of Figures}

3.1 Schematic of wind tunnel experimental setup . . . . . . . . . . 11

$3.2 \quad$ Porous Disk Model . . . . . . . . . . . . . . . . . . . . . 12

3.3 Wind tunnel Passive Spray Grid _ . . . . . . . . . . . . . . . . . . 12

3.4 Porous Disk Front and Side View . . . . . . . . . . . . . . . . . . 12

3.5 Normalized power spectral density (PSD) as a function of wave number $\kappa$ at different free stream tunnel speeds from hotwire measurements for (a) background flow, (b) 1D, and (c) 9.6D wake locations. The $k^{-5 / 3}$ line represents the Kolmogorov spectrum. . . . . . . . . . . . . . 15

4.1 Turbulence intensity $\sigma_{u} / \bar{U}$ vs. mean velocity $\bar{U}$ for the background flow and disk wakes at $1 \mathrm{D}$ and $9.6 \mathrm{D}$ downstream for $U_{\infty}=1.3,4.9,8.3$, $10.6,12.0$, and $15.8 \mathrm{~ms}^{-1} \ldots \ldots \ldots \ldots \ldots$

4.2 Taylor scale Reynolds number $R e_{\lambda}$ as a function of local mean velocity $\bar{U}$ for the background flow and wake at $9.6 \mathrm{D}$ at tunnel speeds $U_{\infty}=1.3$, $4.9,8.3,10.6,12.0$, and $15.8 \mathrm{~ms}^{-1} \ldots \ldots \ldots 21$

4.3 Transverse Taylor microscale $\lambda$, as a function of the Taylor scale Reynolds number $R e_{\lambda}$ for the background flow and the wake at $9.6 \mathrm{D}$ at tunnel speeds $U_{\infty}=1.3,4.9,8.3,10.6,12.0$, and $15.8 \mathrm{~m} \mathrm{~s}^{-1} \ldots \ldots 22$ 
4.4 The Kolmogorov length scale $\eta$ vs. the Taylor scale Reynolds number $R e_{\lambda}$ for the background flow and the wake at 9.6D at tunnel speeds $U_{\infty}=1.3,4.9,8.3,10.6,12.0$, and $15.8 \mathrm{~ms}^{-1} \ldots \ldots 23$

4.5 The integral length scale $L_{\text {int }}$ vs. the Taylor scale Reynolds number $R e_{\lambda}$ for the background flow and the wake at $9.6 \mathrm{D}$ at tunnel speeds $U_{\infty}=$ $1.3,4.9,8.3,10.6,12.0$, and $15.8 \mathrm{~ms}^{-1} \ldots \ldots \ldots \ldots$

4.6 $C_{\varepsilon}$ and $L_{\text {int }} / \lambda$ are plotted vs. the Taylor scale Reynolds number $R e_{\lambda}$ for the background flow and the wake at $9.6 \mathrm{D}$ at tunnel speeds $U_{\infty}=1.3$, $4.9,8.3,10.6,12.0$, and $15.8 \mathrm{~ms}^{-1} \ldots \ldots \ldots \ldots$

4.7 PDF of particle counts as a function of stream-wise velocity for one Reynolds number. Two volume fractions, two wake locations (1D and 9.6D) and wake vs. background flow are compared. . . . . . . . . .

4.8 PDF of particle counts as a function of particle diameter for wakes at $1 \mathrm{D}$ and $9.6 \mathrm{D}$ (rows), and water flow rates of 1.7 and $2.0 \mathrm{Lmin}^{-1}$ (columns) for three different Reynolds numbers, and background flows

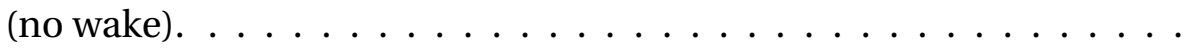


4.9 Normalized mean streamwise velocity $\bar{u} / U_{\infty}$ and mean vertical velocity $\bar{v} / U_{\infty}$ for single-phase flow and two-phase particle velocity fields with increasing volume fractions for $R e_{\lambda}=88.7$. The flow is from the left and column pairs represent the near and far wake regions of interest. Row $\Phi_{0}$ represents single-phase flow, while $\Phi_{1.2}, \Phi_{1.7}$, and $\Phi_{2}$ represent two-phase flow with water flow rates of 1.2, 1.7, and $2 \mathrm{Lmin}^{-1}$ and volume fractions of $4.26 \times 10^{-6}, 6.03 \times 10^{-6}$, and $7.09 \times 10^{-6}$ respectively. Note that the near wake (left) and far wake (right) columns have different scales so that spatial features can be identified. . . . . . . .

4.10 Normalized mean Reynolds stresses $\overline{u^{\prime} u^{\prime}} / U_{\infty}^{2}, \overline{u^{\prime} v^{\prime}} / U_{\infty}^{2}$, and $\overline{v^{\prime} v^{\prime}} / U_{\infty}^{2}$ for single-phase flow and two-phase particle velocity fields with increasing volume fractions for $R e_{\lambda}=88.7$. The flow is from the left and column pairs represent the near and far wake regions of interest. Row $\Phi_{0}$ represents single-phase flow, while $\Phi_{1.2}, \Phi_{1.7}$, and $\Phi_{2}$ represent twophase flow with water flow rates of $1.2,1.7$, and $2 \mathrm{Lmin}^{-1}$ and volume fractions of $4.26 \times 10^{-6}, 6.03 \times 10^{-6}$, and $7.09 \times 10^{-6}$ respectively. Note that the near wake (left) and far wake (right) columns have different scales so that spatial features can be identified. . . . . . . . . . .

4.11 Normalized mean turbulence statistics as a function of height at 1D (solid lines) and 9.6D (dashed lines). $\Phi_{0}$ represents single-phase flow, while $\Phi_{1.2}, \Phi_{1.7}$, and $\Phi_{2}$ represent two-phase flow with water flow rates of 1.2, 1.7, and $2 \mathrm{Lmin}^{-1}$ and volume fractions of $4.26 \times 10^{-6}, 6.03 \times 10^{-6}$, and $7.09 \times 10^{-6}$ respectively. Flow direction is from the left, and $\mathrm{y} / \mathrm{D}=0$ is the center of the disk. . . . . . . . . . . . . . . 
4.12 Zoomed in normalized mean turbulence statistics as a function of height at 9.6D. $\Phi_{0}$ represents single-phase flow, while $\Phi_{1.2}, \Phi_{1.7}$, and $\Phi_{2}$ represent two-phase flow with water flow rates of $1.2,1.7$, and $2 \mathrm{~L} \mathrm{~min}^{-1}$ and volume fractions of $4.26 \times 10^{-6}, 6.03 \times 10^{-6}$, and $7.09 \times 10^{-6}$ respectively. Flow direction is from the left, and $y / D=0$ is the center of the disk. ....................... 36 


\section{Chapter 1}

\section{Introduction}

This study explores the behaviour of inertial particles (water droplets) in the wake behind a stationary porous disk in homogeneous isotropic turbulence (HIT). Many flows of interest in the natural environment and engineering applications are turbulent. Turbulent wakes are of interest where re-circulation, entrainment, energy deficits, and pressure fluctuations affect the design and efficiency of wake-creating bodies such as wind turbines, aircraft, buildings, and bridges. The influence of wakes behind forests, mountains, and islands are also of interest in the natural sciences. The combination of inertial particles in turbulent flows are involved in air pollution, cloud formation, snow, fog, and rain. Raindrop fall speeds are essential for efficient rain collection and prevention of soil erosion.

The study of turbulent two-phase flows has applications in aerospace combustion propulsion systems where liquid fuel is injected as a spray of small droplets in a combustion chamber. Flame ignition and stability depend on the droplet dynamics where the liquid jet is atomized into small droplets that are carried by the gas flow.

Atomizers are also used in industrial processes, including coating applications, cooling towers, and inkjet printing. Chemical fire suppressants depend on the dispersal of droplets that either travel along trajectories, move with the convecting flow, 
or impact upon nearby solid obstacles. To prevent herbicide drift, Soto et al. [23] atomized larger droplets directly above plants with a mesh screen, as smaller droplets more effectively adhere to the leaves, but larger droplets are less likely to be carried downwind.

The development of aerosol removal from gas streams in indoor environments with HVAC or filtration systems is a crucial step in stopping the airborn transmission of diseases. These flows are usually turbulent and filtration involves porous media or impaction collectors, while another option is to direct the particles past microbe killing UV lamps. Evidence has shown the collection efficiency of impaction collectors depends on the ratio of particle stop distance to collector diameter. [12]

Wind tunnel experiments investigating the transport processes of wakes behind solid disks and other bluff bodies have been conducted for several decades. Hwang et al. [11] performed wind tunnel experiments to investigate the decay of turbulence in axisymmetric turbulent wakes behind flat circular disks and observed three discernible wake regions; a near wake region with highly anisotropic turbulence, a similarity region $(100<x / D<400)$ where isotropic turbulence relations were adequate for estimating decay, and a highly intermittent far wake region. Humphries and Vincent [10] studied the recirculation zone in the near wake of flat disks and developed a free stream turbulence parameter $\Lambda=l_{f} k_{f}^{\frac{1}{2}} / D U$, where $l_{f}$ and $k_{f}$ are the length scale and kinetic energy of the free stream turbulence respectively. Works by Townsend and George [27] [8] established foundations for classical turbulence theory of free-shear flows.

Johansson and George [13] carried out a proper orthogonal decomposition study on hot-wire data from the axisymmetric turbulent wake behind a solid disk from 10 
to 50 disk diameters downstream with a Reynolds number of $28 \times 10^{3}$. In another experiment, they investigated the far wake from 10 to 150 disk diameters downstream with a Reynolds number of $26.4 \times 10^{3}$ and concluded their data agreed with the highReynolds-number equilibrium similarity solution for the wake growth. [14]

Dairay et al. [7] completed direct numerical simulations (DNS) and hot wire anemometry (HWA) experiments to confirm assumptions made by Nedić et al. [18] who postulated there are turbulent wake regions where the mean flow velocity deficit decays as the inverse of the distance from the wake-generating body and the wake width grows as the square root of the that distance.

For some time, the wakes behind porous disks have been studied as analogs to more complicated wakes. Roberts [20] studied drag coefficients of solid and porous disks with open area ratios of 2-33 percent for parachute applications. Aubrun et al. [3] compared wake properties of a porous disk to a rotating wind turbine model in wind tunnel experiments with two different inflow conditions: isotropic turbulent inflow, and a neutral atmospheric boundary layer. They identified that while the wakes are indistinguishable after three disk diameters downstream in the atmospheric boundary layer case, there are still discrepancies in the isotropic turbulent inflow case.

Aloui et al. [2] compared PIV measurements of steady and unsteady wakes behind porous disks. Their POD analysis showed that alternating vortices form in the unsteady wake. Lignarolo et al. [15] presented an experimental analysis comparing the near wakes of a wind turbine model and porous disk. Their results establish a good match between the turbine and the disk for velocity, pressure, and enthalpy fields, but show differences in turbulence intensity and turbulent mixing. Camp and Cal [6] compared an array of porous disks to an array of rotating turbines and quantified the dif- 
ferences in the mean kinetic energy transport within the wakes. They ascertained the primary difference was in the spanwise mean velocity component in the near wake region. Naderi and Torabi [17] ran numerical simulations of porous disks as analogs to rotating wind turbines and tested different turbulence models, showing the nacelle had a significant effect on the downstream wake and should be taken into account.

Others have studied inertial particles in turbulent flows. Toschi and Bodenschatz, Balachandar and Eaton, and Bourgoin and Xu provide good overviews of Lagrangian particles in turbulence in the following review papers. Toschi and Bodenschatz focus on tracer and particle dynamics and compare computational techniques to experimental data. [26] Blachandar and Eaton describe experimental and computational techniques for turbulent, dispersed, multiphase flows including preferential concentration, coupling between the two-phases, and turbulence modulation. [4] Bourgoin and $\mathrm{Xu}$ highlight some of the latest developments in numerical simulations, theory, and experimental methods. [5]

Aliseda et al. and Good et al. [1] [9] investigated the settling velocities of inertial particles in HIT with experiments and direct numerical simulations (DNS). Aliseda et al. found the particle settling velocity to be much larger than quiescent fluid, and enhancement of the settling velocity increases with volume fraction, while Good et al. found regions of high velocity r.m.s. anisotropy generally coincide with regions of settling velocity reductions. Sumbekova et al. and Obligado et al. [24] [19] studied preferential concentration of inertial particles in HIT. Sumbekova et al. and determined particles within clusters settle faster than particles in voids. Obligado et al. found cluster settling velocity has a strong dependence on $R e_{\lambda}$ and may be linked to settling velocity enhancement/hindering due to the carrier turbulence Rouse number (ratio of particle 
terminal velocity in still fluid to standard deviation of turbulent velocity fluctuations) or turbulence acceleration ratio, which is the ratio of the standard deviation of fluid acceleration fluctuations to gravity. They show that clustering increases with both $\phi_{v}$ and $R e_{\lambda}$. They find that the mean size of clusters increases with $R e_{\lambda}$ but decreases with $\phi_{v}$

The axisymmetric turbulent wake is still an unsolved problem in physics where even the mean scales are still being discussed. There are also questions on the role of coherent structures and entrainment. These questions become more complex when the carrier flow is coupled with inertial particles. One must consider settling velocity modification and clustering.

As this is a first study on the subject, the focus is on the homogeneous isotropic turbulence incoming flow case. The persistence of the wake, how particles are entrained, particle discrimination by size, and particle settling velocity are studied. To the author's knowledge, this is the first experimental study on the coupling of inertial particles with a self-similar, large $\mathrm{Re}_{\lambda}$ flow. Theory is presented in Chapter 2 , the experimental setup and data collection techniques are presented in Chapter 3, and results are presented in Chapter 4. The application of the analysis techniques and discussion follow in Chapter 5, and concluding remarks on the implications are given in Chapter 6. 


\section{Chapter 2}

\section{Theory}

\subsection{Turbulence Statistics}

Time averaged turbulence statistics are based on the Reynolds Averaged Navier-Stokes (RANS) equation for incompressible flow:

$$
\overline{u_{j}} \frac{\partial \overline{u_{i}}}{\partial x_{j}}=-\frac{1}{\rho} \frac{\partial \bar{p}}{\partial x_{i}}-\frac{\partial\left(\overline{u_{i}^{\prime} u_{j}^{\prime}}\right)}{\partial x_{j}}+\overline{f_{i}}
$$

where $u$ is carrier fluid velocity, $x$ is the direction in three dimensional space, $p$ and $\rho$ are the carrier fluid pressure and density, and $f$ represents forces on the carrier fluid from particle interactions. Subscripts $i$ and $j$ are indices where $x_{1}, x_{2}, x_{3}=$ the $x, y, z$ directions in three dimensional space representing the streamwise, vertical, and spanwise directions respectively. Overbars represent time-averaged quantities and prime symbols represent fluctuating quantities. Instantaneous velocity signals are decomposed with the Reynolds decomposition as:

$$
u(x, y, z, t)=\overline{u(x, y, z)}+u^{\prime}(x, y, z, t)
$$


It is assumed that viscous stresses do not significantly affect the mean flow and the majority of stress is from the momentum flux, otherwise known as the Reynolds stresses:

$$
\frac{\partial\left(\overline{u_{i}^{\prime} u_{j}^{\prime}}\right)}{\partial x_{j}}
$$

Other turbulence quantities calculated in this study include the turbulent dissipation rate and turbulence length scales calculated from hot-wire anemometry measurements using Taylor's hypothesis; which approximates spatial correlations by temporal correlations and can be applied to HIT where $u^{\prime} / \bar{u} \ll 1$. The turbulent dissipation rate can be calculated by $\varepsilon=\int 15 v k_{1}^{2} E_{11} d k_{1}$ where $E_{11}$ is the energy spectrum function and $k_{11}$ is the wave number of the fluctuating velocity signal. The turbulence length scales are as follows:

The integral length scale, which is the size of the largest turbulent eddies:

$$
L_{\text {int }}=\int_{0}^{\infty} \rho(r) \mathrm{d} r
$$

where $\rho(r)$ is the longitudinal auto-correlation function of the velocity signal.

The Taylor micro-scale, which represents the intermediate length scale at which fluid viscosity significantly affects turbulent eddies in the flow:

$$
\lambda=\sqrt{\left(15 v\left(\sigma_{u}\right)^{2}\right) / \varepsilon}
$$

Where $v$ is the kinematic viscosity of air and $\sigma_{u}$ is the standard deviation of stream wise velocity fluctuations.

The Kolmogorov length scale, which is the scale of the smallest eddies where kinetic 
energy is dissipated as heat:

$$
\eta=\left(v^{3} / \varepsilon\right)^{1 / 4}
$$

From these quantities the Taylor scale Reynolds number can be found:

$$
R e_{\lambda}=\frac{\sigma_{u} \lambda}{v}
$$

Kolmogorov's 1941 theory (K41) states that:

$$
\varepsilon=C_{\varepsilon} \frac{\sigma_{u}^{3}}{L_{\mathrm{int}}}
$$

where $C_{\varepsilon}$ is the kinetic energy dissipation constant, and is linear in the inertial range. $C_{\varepsilon}$ is not constant under certain conditions and is discussed further in Vassilicos [28].

\subsection{Multiphase flow}

For the two-phase flow cases, inertial particle (water droplet) volume fractions were calculated as $\Phi_{v}=Q_{w} /\left(Q_{w}+Q_{a}\right)$ where $Q_{w}$ and $Q_{a}$ are water and air volumetric flow rates in the wind tunnel.

The Stokes number, which is the ratio of particle viscous relaxation time $\left(\tau_{p}\right)$ and the Kolmogorov time scale $\left(\tau_{\eta}\right)$ for a particle is defined as:

$$
S t=\frac{\tau_{p}}{\tau_{\eta}}=\frac{\rho_{p} D^{2} \varepsilon^{1 / 2}}{18 \rho_{f} v^{3 / 2}}
$$

where $\rho_{p}$ and $\rho_{f}$ are the particle and carrier fluid densities. $D$ is the average particle diameter. Particle clustering and transition from one-way coupled (where particles do 
not affect the flow) to two-way coupled (where the particles and the flow affect each other) flow is dependent on the Stokes number.

Lagrangian motion of inertial particles in turbulence can be modeled as [21]:

$$
\begin{gathered}
\frac{\mathrm{d} \mathbf{V}(t)}{d t}=-f\left(R e_{\mathrm{p}}, V_{\mathrm{rel}}\right) \frac{\mathbf{V}(t)-\mathbf{U}(\mathbf{Y}(t), t)}{\tau_{p}}+\mathbf{g} \\
\frac{d \mathbf{Y}(t)}{d t}=\mathbf{V}(t)
\end{gathered}
$$

Where $\mathbf{V}(t)$ is the particle velocity, $\mathbf{U}(\mathbf{X}, t)$ is the background turbulent velocity, and $\mathbf{U}(\mathbf{Y}(t), t)$ is the fluid velocity at the particle location $\mathbf{Y}(t) . \tau_{p}$ is the particle viscous relaxation time, $R e_{\mathrm{p}}$ is the particle Reynolds number, $V_{\text {rel }}$ is particle-fluid relative velocity, and $\mathbf{g}$ is particle acceleration. 


\section{Chapter 3}

\section{Experimental Setup}

Experiments were conducted at the Université Grenoble Alpes at LEGI laboratory in the Lespinard Wind Tunnel. The closed-circuit wind tunnel test section is $4 \mathrm{~m}$ long with a cross sectional area of $0.75 \mathrm{~m} \times 0.75 \mathrm{~m}$ as shown in figure 3.1. figure 3.3 shows the passive spray grid with 36 water misting nozzles with $0.4 \mathrm{~mm}$ diameter at the tunnel inlet that inject inertial water droplets into the tunnel flow. A stationary open grid is located $15 \mathrm{~cm}$ upstream of the spray grid to compensate and mix the turbulence added by the injectors, and the combined grids produce turbulence on the order of $2.4-3.0 \%$. A 150 bar pump with variable flow rate control, supplied water to the spray grid, producing a uniform spray of poly-disperse water droplets with average diameter of $43 \mu \mathrm{m}$. Water flow rate was controlled with a manual variable regulator and data was collected after the water flow-rate, humidity, and tunnel velocity reached steady state.

A porous disk attached to a $12.7 \mathrm{~mm}$ diameter aluminum tube was mounted in the wind tunnel and attached at the tunnel floor as shown in Figure 3.1. The disk has a diameter of $12 \mathrm{~cm}$, a thickness of $3.175 \mathrm{~mm}$, a porosity of approximately $56 \%$, and a tunnel blockage ratio of $1.57 \%$ including the mounting tube. A small blockage ratio allows unimpeded expansion of the wakes within the tunnel. Refer to Figures 3.2 and 3.4 for disk geometry. Further information on the disc design and dimensions can be 


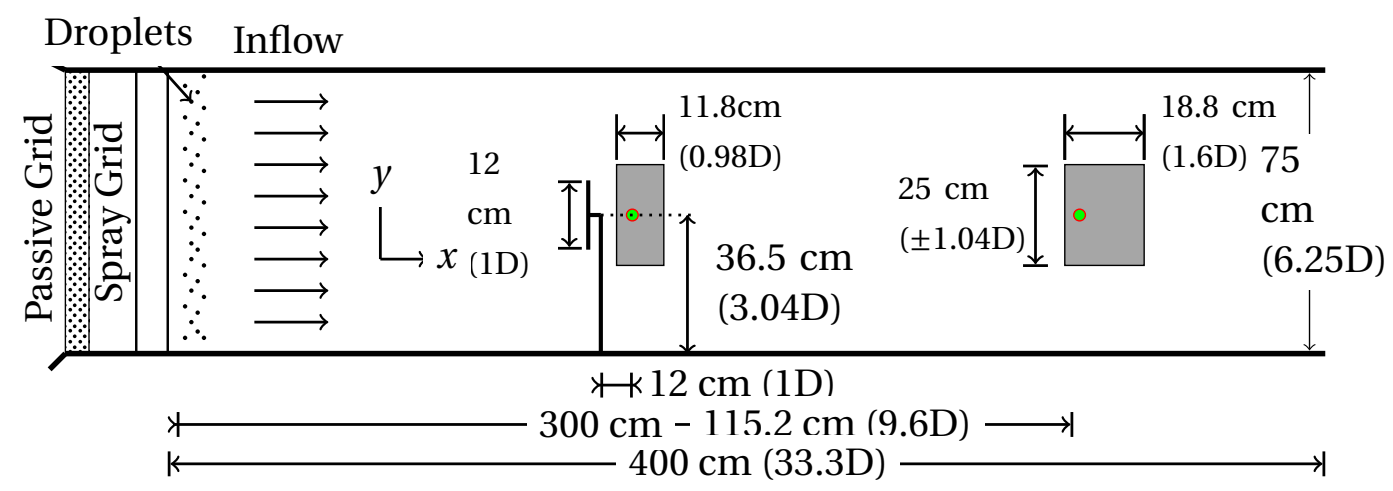

Figure 3.1: Schematic of wind tunnel experimental setup, side view. (Schematic is not to scale) Note that the measurement location is fixed at $300 \mathrm{~cm}$ downstream of the grid and the disk model was moved for near and far wake measurements. The height of the measurement windows is 2.08D. Image provided by Smith et al. [22]

found in Camp and Cal [6].

Hot-wire anemometry, phase Doppler interferometry (PDI) and particle image velocemitry (PIV) measurements were taken of the background flow (no wake) and compared to locations at one disk diameter (1D) and 9.6 disk diameters (9.6D) downstream of the disk wake to analyze characteristics of different flow regimes. The disk was positioned in the center of the tunnel cross section for the hot-wire anemometry and PDI measurements, and $20 \mathrm{~cm}$ from the tunnel wall for the PIV measurements. The offcenter location of the disk for PIV measurements was due to hardware mounted to the top outer side of the tunnel preventing a central placement of the laser optic.

\subsection{Inflow Characterization}

The inflow conditions without inertial particles were characterized with hotwire anemometry measurements using a Dantec Streamline constant temperature anemometer with a tungsten wire probe. The Pt-W 55P01 type probe had a sensing 


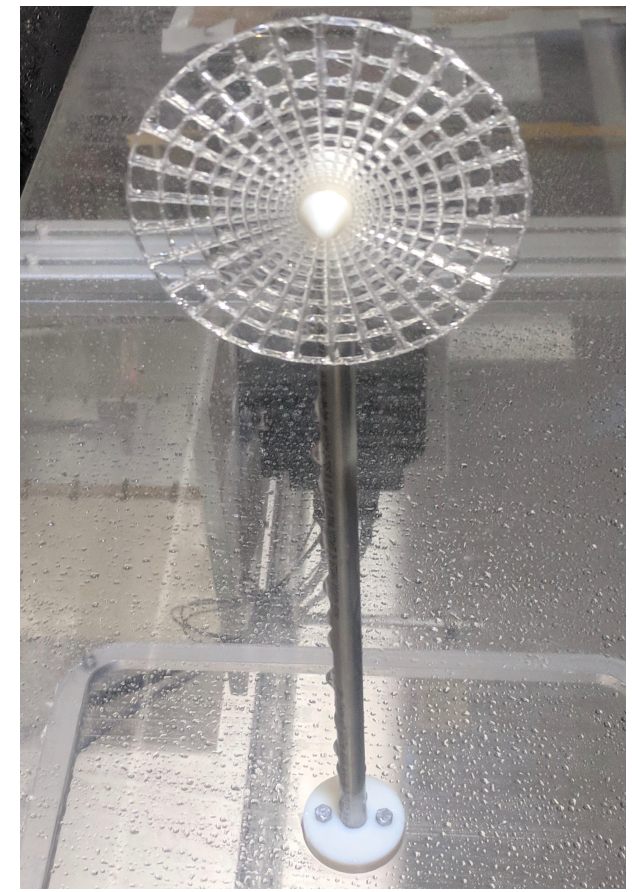

Figure 3.2: Porous Disk Model

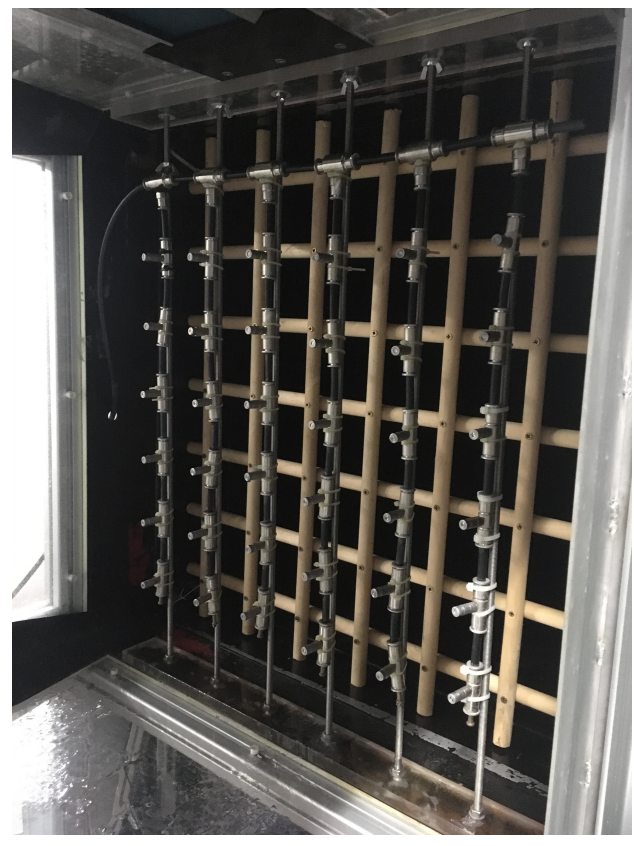

Figure 3.3: Wind tunnel Passive Spray Grid
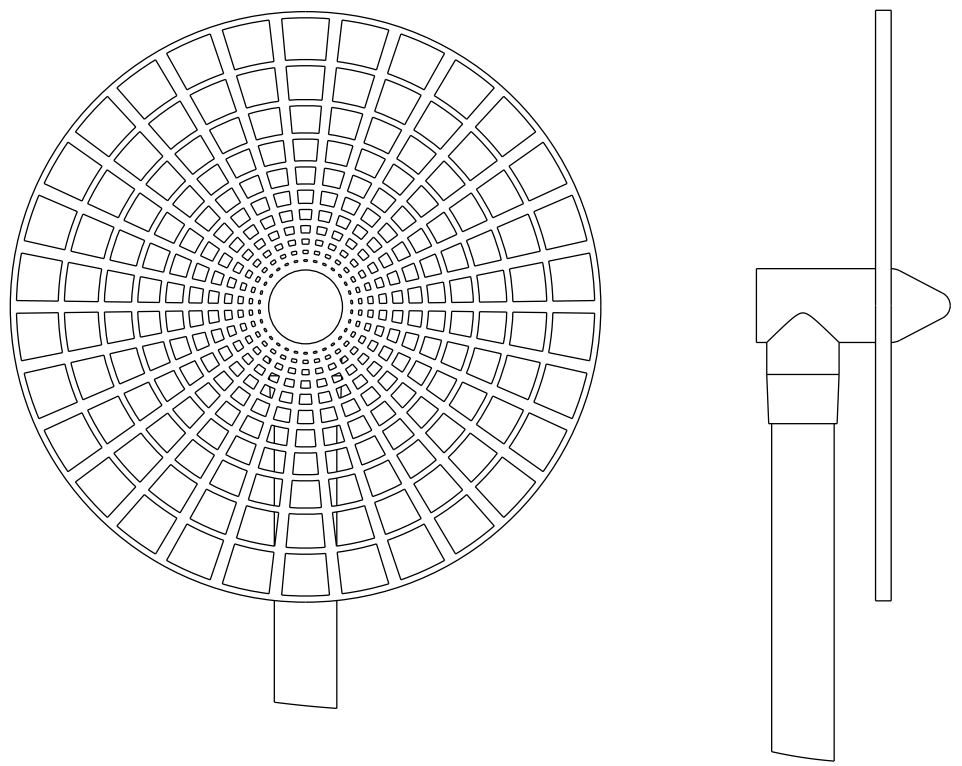

Figure 3.4: Porous Disk Front and Side View 
length of $1.25 \mathrm{~mm}$ and a wire diameter of $5 \mu \mathrm{m}$. Data acquisition time was 300 seconds at either $20 \mathrm{kHz}$ or $35 \mathrm{kHz}$ at constant temperature and pressure, and velocities were calculated using King's law and Taylor's hypothesis. Adequate resolution was achieved to resolve $\eta$ as $\kappa \eta \geq 1$, where $\kappa$ is the wave number $\left[\mathrm{radm}^{-1}\right]$ and $\eta$ is the Kolmogorov length scale $[\mathrm{m}]$.

To quantify turbulence in the absence of particles, the hot-wire was aligned vertically with the center of the disk at $36.5 \mathrm{~cm}$ from the tunnel wall and floor. Measurements were taken in the open tunnel at $3 \mathrm{~m}$ downstream of the grid, and in the near and far wakes at $1 \mathrm{D}$ and $9.6 \mathrm{D}$ respectively for tunnel speeds $U_{\infty}=2.6,4.9,8.4,10.6$, 12.0 , and $15.8 \mathrm{~ms}^{-1}$.

Turbulence statistics were calculated for each wake location and tunnel speed. Table 3.1 presents the disk diameter Reynolds number $R e_{D}=U_{\infty} D / v$, the Taylor scale Reynolds number $R e_{\lambda}=\sigma_{u} \lambda / v$, the integral length scale $L_{\text {int }}=\int_{0}^{\infty} \rho(r) d r$, the Taylor micro-scale $\lambda=\sqrt{\left(15 v\left(\sigma_{u}\right)^{2}\right) / \varepsilon}$, the Kolmogorov length scale $\eta=\left(v^{3} / \varepsilon\right)^{1 / 4}$, and the kinetic energy dissipation equation constant $C_{\varepsilon}=\varepsilon L_{\mathrm{int}} / \sigma_{u}^{3}$ in the background flow (no wake) for each free stream velocity at $x=1 \mathrm{~m}$. For the following equations, $U_{\infty}$ is the free stream velocity, $D$ is the disk diameter, $v$ is the kinematic viscosity of air, $\sigma_{u}$ is the standard deviation of stream wise velocity, $\rho(r)$ is the longitudinal auto-correlation function, and $\varepsilon$ is the turbulent dissipation rate calculated based on Taylor's hypothesis.

Figure 3.5 shows calculated normalized power spectral density (PSD) as a function of wave number $\kappa$ for the measured instantaneous velocities for the background flow and disk wake locations at $1 \mathrm{D}$ and 9.6D downstream. The presence of the wake increases the wave number, with the near wake at $1 \mathrm{D}$ downstream having the greatest 


\begin{tabular}{r|llllll}
$\mathbf{U}_{\infty}\left[\mathrm{m} \mathrm{s}^{-1}\right]$ & 2.61 & 4.86 & 8.35 & 10.6 & 12.0 & 15.8 \\
\hline $\mathbf{R e}_{\mathbf{D}}\left(\times 10^{4}\right)$ & 2.02 & 3.75 & 6.51 & 8.16 & 9.38 & 12.2 \\
$\mathbf{R e}_{\lambda}$ & 48.9 & 66.9 & 88.7 & 102 & 99.7 & 120 \\
$\mathbf{L}_{\mathrm{int}}[\mathrm{mm}]$ & 37.3 & 33.5 & 34.9 & 34.2 & 31.4 & 33.0 \\
$\boldsymbol{\lambda}[\mathrm{mm}]$ & 11.0 & 8.33 & 6.72 & 6.06 & 5.35 & 4.91 \\
$\boldsymbol{\eta}[\mu \mathrm{m}]$ & 798 & 518 & 363 & 305 & 273 & 227 \\
$\mathbf{C}_{\varepsilon}$ & 1.04 & 0.901 & 0.878 & 0.831 & 0.883 & 0.893
\end{tabular}

Table 3.1: Calculated Turbulence Statistics for the Background Flow

wave number. This indicates the wakes are producing smaller eddies and increased energy dissipation than that of the background flow. It is also interesting to note the signals with slope closest to the Kolmogorov-based inertial range $\left(k^{-5 / 3}\right)$ are at $9.6 \mathrm{D}$ in the far wake.

\subsection{Two-phase Flow Experiments}

Table 3.2 lists case parameters for both the PDI and PIV measurements. Water was delivered to the spray grid at $1.2,1.7$, and $2.0 \mathrm{Lmin}^{-1}$ at three different free stream velocities resulting in various water volume fractions $\Phi_{\nu}$. The Stokes number $S t=\tau_{p} / \tau_{\eta}=$ $\left(\rho_{p} D^{2} \varepsilon^{1 / 2}\right) /\left(18 \rho_{f} v^{3 / 2}\right)$ based on a probable particle diameter $41 \mu \mathrm{m}$ (as shown in Figure 4.8) was calculated for the background flow (no wake), and the minimum and maximum water volume fractions are listed for each velocity.

\subsubsection{PDI}

The PDI measurement location is denoted with small green circles in Figure 3.1 and was located $3 \mathrm{~m}$ downstream of the grid, $36.5 \mathrm{~cm}$ from the floor and receiver side of tunnel, and $38.5 \mathrm{~cm}$ from the laser transmitter side. The PDI system remained station- 

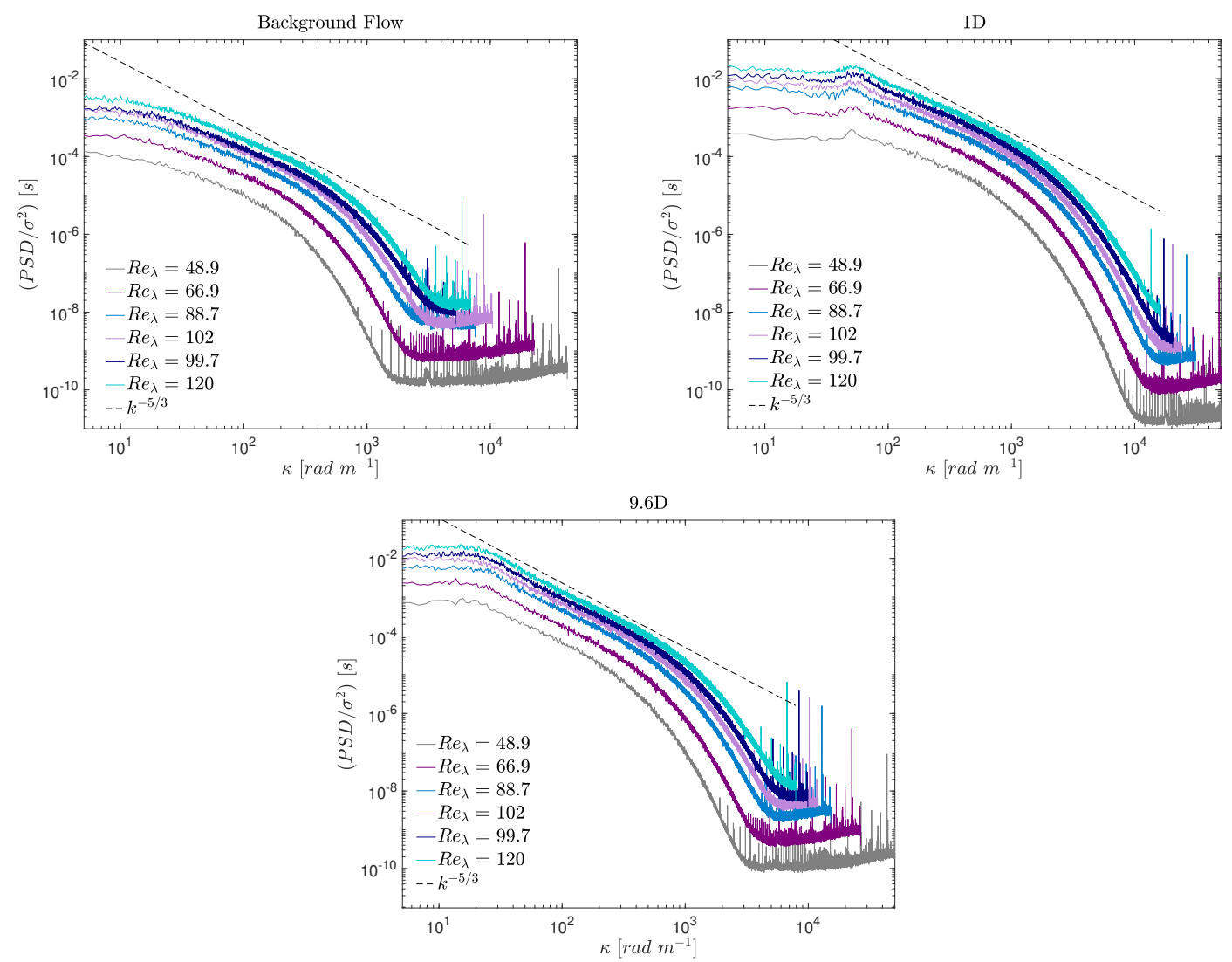

Figure 3.5: Normalized power spectral density (PSD) as a function of wave number $\kappa$ at different free stream tunnel speeds from hotwire measurements for (a) background flow, (b) 1D, and (c) 9.6D wake locations. The $k^{-5 / 3}$ line represents the Kolmogorov spectrum.

ary while the disk was positioned in the tunnel such that the PDI measurement location was centered behind the disk at 1D and 9.6D downstream. PDI data was collected with an Artium Technologies PDI-200MD system, capable of detecting particle diameter ranges from 0.3 - $800 \mu \mathrm{m}$ with a dimensional accuracy of $\pm 0.5 \mu \mathrm{m}$ and a velocity range of -600 to $1000 \mathrm{~ms}^{-1}$ with $\pm 1 \%$ accuracy. Two diode pumped solid state lasers with wavelengths of 532 and $492 \mathrm{~nm}$ were split into two beams of equal intensity with the $532 \mathrm{~nm}$ beam oriented to measure vertical velocity and the $491 \mathrm{~nm}$ beam oriented 


\begin{tabular}{r|lll}
$\mathbf{U}_{\infty}\left[\mathrm{m} \mathrm{s}^{-1}\right]$ & 4.86 & 8.35 & 12.0 \\
\hline $\mathbf{R e}_{\mathbf{D}}\left(\times 10^{4}\right)$ & 3.75 & 6.51 & 9.38 \\
St (Background Flow) & 0.285 & 0.581 & 1.03 \\
$\operatorname{Min} \Phi_{\mathbf{v}}\left(\times 10^{-6}\right)$ & 7.31 & 4.26 & 2.95 \\
$\operatorname{Max} \Phi_{\mathbf{v}}\left(\times 10^{-6}\right)$ & 12.2 & 7.09 & 4.92
\end{tabular}

Table 3.2: PDI and PIV Case Parameters

to measure horizontal velocity. The transmitter and receiver were positioned on opposite sides of the tunnel and had focal lengths of $1000 \mathrm{~mm}$ and $500 \mathrm{~mm}$ respectively. The receiver aperture was $200 \mu \mathrm{m}$. Signals were analyzed with the Artium advanced signal analyzer (ASA) with a maximum sampling frequency of $320 \mathrm{MHz}$ and resolution of 0.01 $\%$ of sampling frequency. $500 \times 10^{3}$ signals were collected per case with the Automated Instrument Management System (AIMS) 5.2 software to assure good statistical convergence. Particle diameters and velocities were measured for water flow-rates of 1.7 and 2.0 $\mathrm{Lmin}^{-1}$ at the free stream tunnel speeds listed in table 3.2.

\subsubsection{PIV}

PIV measurements were taken with all three water flow rates and non-inertial tracer particles $\left(\Phi_{0}\right)$ at a free stream tunnel speed of $8.35 \mathrm{~m} \mathrm{~s}^{-1}$. To prevent laser interference from water droplets adhering to the top inside tunnel window, a $12 \mathrm{~mm}$ angle was attached on the inside upper window upstream of the laser. The vertical laser sheet aligned with the free stream and was centered with respect to the disk giving a velocity field in the $x-y$ plane. Note that the wake is axisymmetric in the $x-z$ plane, but the wake is not symmetric in the $x-y$ plane due to the influence of the tube. The model was painted black to prevent reflections, however, some reflections were still present resulting in a cropped region of interest for the near wake at 1D. Regions of interest for the 
near and far wakes are shown as grey rectangles in Figure 3.1. PIV data was collected with the use of a Litron LD30-527, double pulse, Nd:YLF laser with a frequency of 3.0 $\mathrm{kHz}$ and a wavelength of $527 \mathrm{~nm}$. For non-inertial tracer particles, the flow was seeded with two Antari alpha F-80Z smoke machines with Antari high density smoke Z-Fluid. For inertial particle cases, the inertial particles (water droplets) were used as seeding. A Phantom V2511 high speed camera with a $50 \mathrm{~mm}$ Nikon lens and resolution of 1280 x 800 megapixels was set perpendicular to laser sheet and the camera captured images through an opening in the tunnel wall. The PIV grid size was $0.25 \mathrm{~mm}$ and 8000 pairs of images were analyzed to generate converged turbulence statistics. It was found from approximately $10 \times 10^{3}$ snapshots that that the number of snapshots needed for statistical convergence was below 2000. Non-inertial particle measurements were analyzed with Dantec Dynamic Studio 9.7 PIV software, and inertial particle measurements were analyzed with PIVLab, an open source software by Thielicke and Stamhuis [25]. 


\section{Chapter 4}

\section{Results}

\subsection{Hot-wire Anemometry}

All hot-wire anemometry results are plotted for tunnel speeds $U_{\infty}=4.9,8.3,10.6,12.0$, and $15.8 \mathrm{~ms}^{-1}$. Figure 4.1 shows turbulence intensity vs. the local mean stream-wise velocity $\bar{U}$ for the background single-phase flow and wake locations at $1 \mathrm{D}$ and $9.6 \mathrm{D}$ downstream of the disk. The local mean velocity $\bar{U}$ is reduced by about $54 \%-66 \%$ in the near wake at $1 \mathrm{D}$ and $7 \%-19 \%$ in the far wake at 9.6D. The turbulence intensity increases from 205 - $246 \%$ for the far wake and 869 - $987 \%$ in the near wake. Turbulence intensity remains fairly constant for the background flow and far wake, and varies with $\bar{U}$ in the near wake, indicating the near wake is a highly turbulent region, and the far wake is more turbulent than the background flow. Figures 4.2 - 4.6 compare turbulence statistics (see table 3.1) for the far wake at 9.6D to the background flow conditions. Although hot-wire data was collected in the near wake at 1D, the wake flow at 1D downstream is not homogeneous isotropic turbulence (HIT) and the Taylor hypothesis does not hold.

Figure 4.2 shows the Taylor scale Reynolds number $R e_{\lambda}$ as a function of local mean

velocity $\bar{U}$. For both cases $R e_{\lambda}$ increases with increasing $\bar{U}$, with larger values and a 
steeper increase for the wake. Although $\lambda$ tends to decrease with increasing $\bar{U}, \sigma_{u}$ is increasing at a greater rate and more so for the wake resulting in increasing $R e_{\lambda}$. This shows that $R e_{\lambda}$ is greater in the wake than the background flow. Figure 4.3 shows the transverse Taylor microscale $\lambda$ as a function of $R e_{\lambda} . \lambda$ represents the intermediate length scale at which viscosity significantly affects turbulent eddies, and values of $\lambda$ are smaller in the wake compared to the background flow, indicating the wake is generating more turbulent dissipation. $R e_{\lambda}$ is greater in the wake due to greater $\sigma_{u}$ of the turbulent signal. In both cases $\lambda$ decreases with increasing $R e_{\lambda}$ as higher incoming velocities are also producing more turbulence.

The Kolmolgorov length scale $\eta$ is shown in Figure 4.4 as a function of $R e_{\lambda} \cdot \eta$ represents the scale of the smallest turbulent eddies where kinetic energy is dissipated as heat. $\eta$ decreases with increasing $R e_{\lambda}$ for both cases as well as $\lambda$. For each $R e_{\lambda}, \eta$ is smaller in the wake as the disk is dissipating more kinetic energy than the background flow. For both $\lambda$ and $\eta$, the background flow has a steeper slope than the wake. $\eta$ appears to approach a constant value as $R e_{\lambda}$ increases.

Figure 4.5 shows the integral length scale $L_{\text {int }}$ (representing the largest eddies in the flow) vs. $R e_{\lambda}$. The background flow shows no trend and a greater variation in eddy size (31.4 - $34.9 \mathrm{~mm})$, where the wake produces a more consistent eddy size (32.2 - 33.0 $\mathrm{mm})$. Figure 4.6 compares the dissipation equation constant $C_{\varepsilon}$ and $L_{\mathrm{int}} / \lambda$ vs. $R e_{\lambda}$. $C_{\varepsilon}$ is larger for the background flow where it ranges from $0.83-1.4$, and is reduced by about half in the wake where it ranges from $0.33-0.41 . L_{\text {int }} / \lambda$ steeply increases with increasing $R e_{\lambda}$ for both cases, due to the decrease in $\lambda$ at higher $R e_{\lambda}$. The linear slopes of $L_{\text {int }} / \lambda$ agree with K41 theory that $L_{\text {int }} / \lambda \sim C_{\varepsilon} R e_{\lambda}$, and that the background flow and far wake have HIT and the Taylor hypothesis holds. 


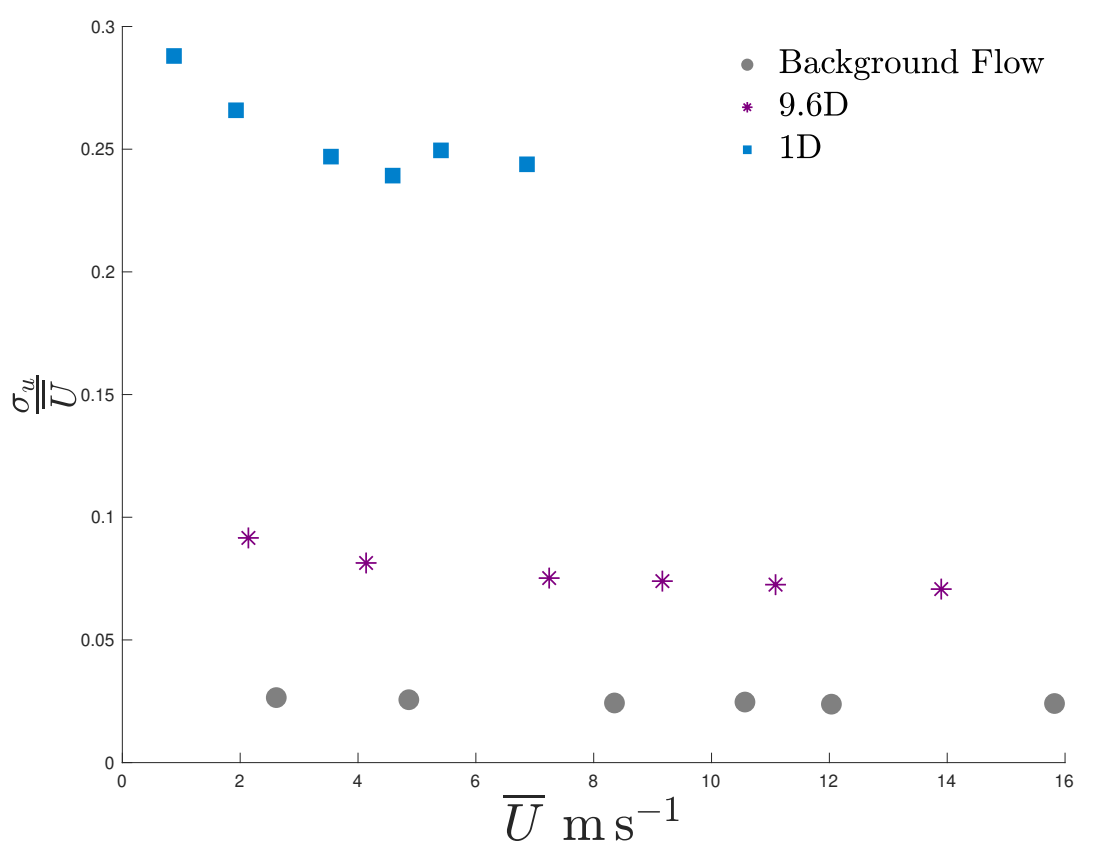

Figure 4.1: Turbulence intensity $\sigma_{u} / \bar{U}$ vs. mean velocity $\bar{U}$ for the background flow and disk wakes at $1 \mathrm{D}$ and 9.6D downstream for $U_{\infty}=1.3,4.9,8.3,10.6,12.0$, and $15.8 \mathrm{~ms}^{-1}$.

Hot-wire anemometry results presented in Figures $4.2-4.6$ show that in the absence of inertial particles, HIT exists in the background flow and far wake; and the Taylor hypothesis holds in the far wake region. The disk produces more turbulence, smaller eddies, and greater dissipation of turbulent kinetic energy than the background flow.

\subsection{PDI}

The following results were obtained using PDI within the wake of the disk and in the background flow. Figure 4.7 shows the probability density function (PDF) of particle counts as a function of particle velocity for near and far wakes at one Reynolds number. 


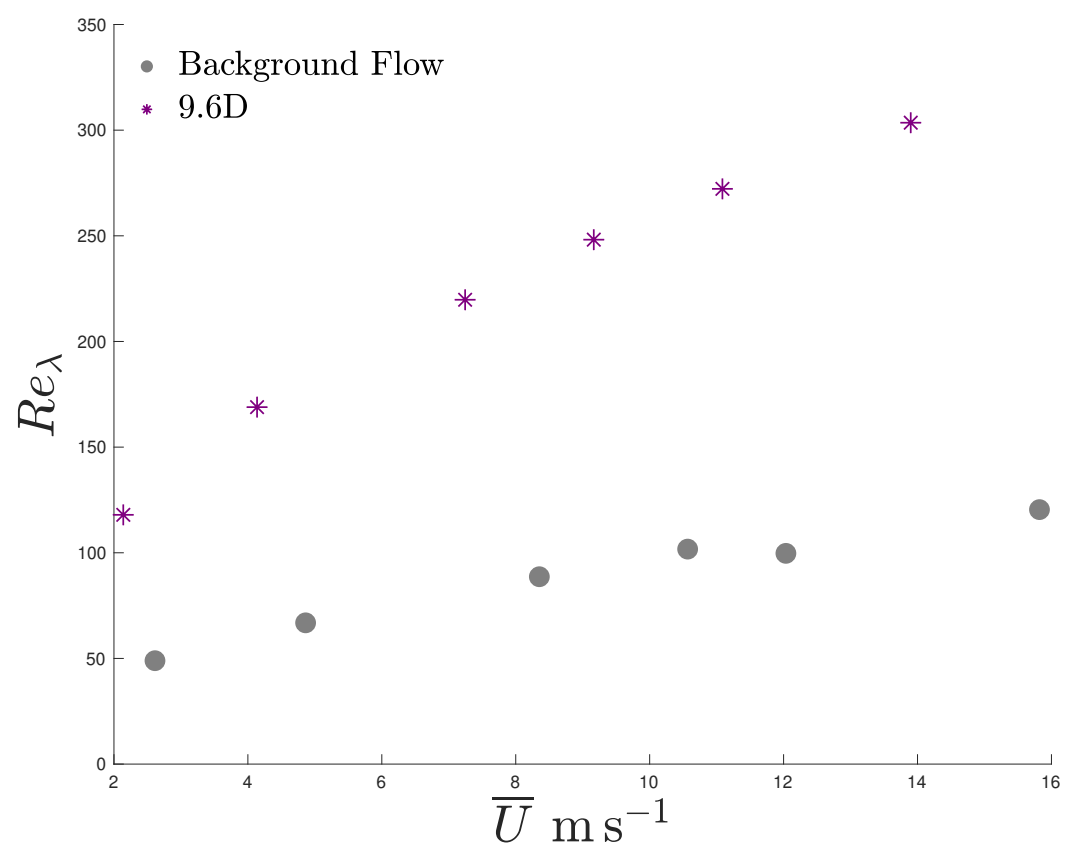

Figure 4.2: Taylor scale Reynolds number $R e_{\lambda}$ as a function of local mean velocity $\bar{U}$ for the background flow and wake at $9.6 \mathrm{D}$ at tunnel speeds $U_{\infty}=1.3,4.9,8.3$, $10.6,12.0$, and $15.8 \mathrm{~ms}^{-1}$.

Two different water volume fractions $\Phi_{1.7}$ and $\Phi_{2}$ represent water volume fractions of $4.26 \times 10^{-6}$ and $6.03 \times 10^{-6}$ respectively, where the subscripts refer to 1.7 and $2.0 \mathrm{Lmin}^{-1}$ water volumetric flow rates.

Particle velocities are reduced in the near wake by approximately $144-141 \%$, and are negative, indicating recirculation. This flow reversal confirms why the hot-wire measurements cannot accurately characterize the near wake at 1D. Particles in the far wake have a velocity reduction of $7.4-12.2 \%$ confirming that the wake is still present at 9.6D downstream. Increasing the water volume fraction reduces the particle streamwise velocities, and is possibly due to larger particle diameters at higher volume fractions shown in Figure 4.8. 


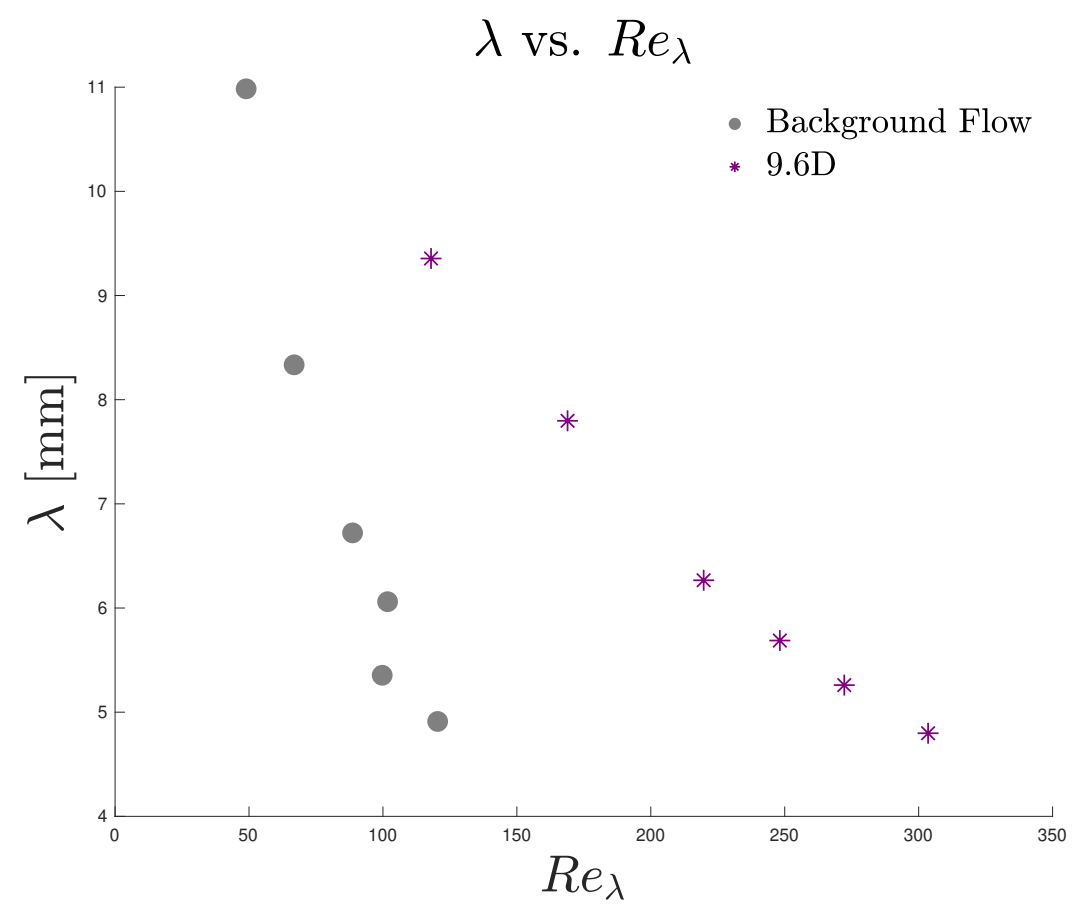

Figure 4.3: Transverse Taylor microscale $\lambda$, as a function of the Taylor scale Reynolds number $R e_{\lambda}$ for the background flow and the wake at $9.6 \mathrm{D}$ at tunnel speeds $U_{\infty}=1.3,4.9,8.3,10.6,12.0$, and $15.8 \mathrm{~m} \mathrm{~s}^{-1}$.

Figure 4.8 shows probability density functions (PDFs) of particle counts as a function of particle diameter for two different water flow rates (columns) and 1D and 9.6D wake locations (rows) for three different Reynolds numbers. Probable particle diameters range from $13 \mu \mathrm{m}$ in the near wake to $41 \mu \mathrm{m}$ in the background flow. For all cases, diameters are 2 - $10 \mu \mathrm{m}$ larger for the higher flow rate, and there is a $3-21 \mu \mathrm{m}$ difference between 1D and 9.6D, with smaller diameters at 1D. At 9.6D particle diameter distributions are similar between the wake and the background flow, with the most probable particle diameter around $30 \mu \mathrm{m}$. In contrast, the wake at 1D produces a much higher probability of particles around $17 \mu \mathrm{m}$, implying preferential trapping of smaller particles within the near wake region. Figures 4.7 and 4.8 illustrate recirculation and 


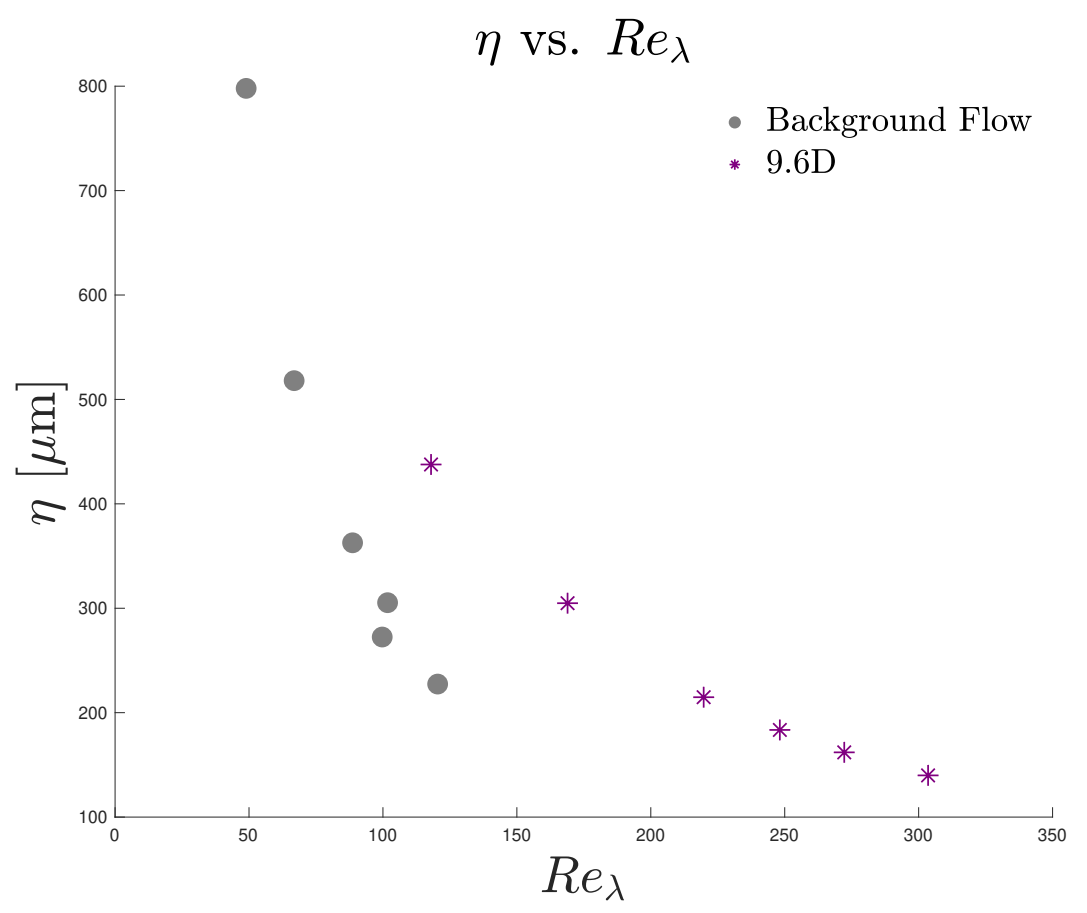

Figure 4.4: The Kolmogorov length scale $\eta$ vs. the Taylor scale Reynolds number $R e_{\lambda}$ for the background flow and the wake at $9.6 \mathrm{D}$ at tunnel speeds $U_{\infty}=1.3,4.9$, $8.3,10.6,12.0$, and $15.8 \mathrm{~m} \mathrm{~s}^{-1}$.

trapping of small particles at $1 \mathrm{D}$, and that the wake still influences the particles at $9.6 \mathrm{D}$ downstream.

\subsection{PIV}

Turbulence statistics calculated from PIV measurements are compared for inertial particles (water droplets) and sub-inertial particles (smoke) in near and far regions of the disk wake. Inertial particles were used as the seeding for PIV measurements to observe particle behaviour. Figures 4.9 and 4.10 show contour plots of various time-averaged normalized statistics at $R e_{\lambda}=88.7$, in areas of interest from $\mathrm{x} / \mathrm{D}=0.75 \mathrm{D}-1.75 \mathrm{D}$ for the near wake, and 9.5D - 11D for the far wake. Row $\Phi_{0}$ shows contour plots of the single- 


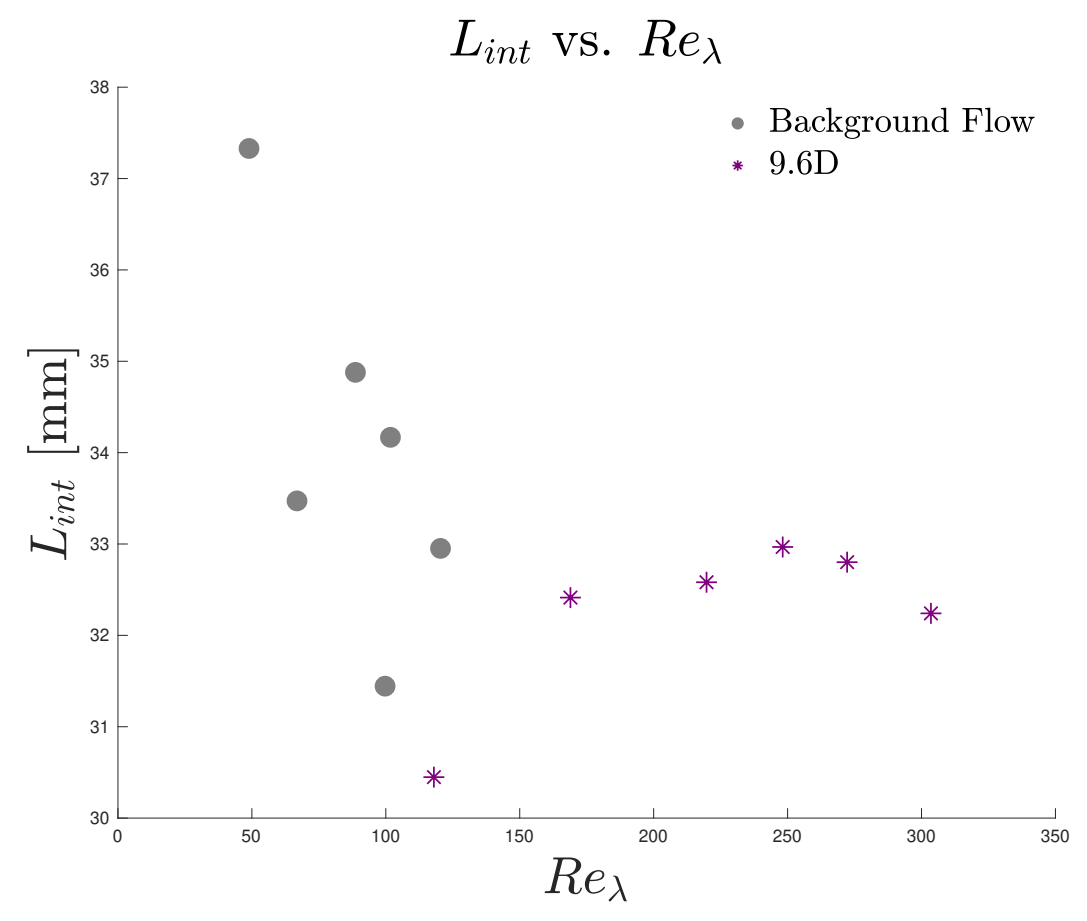

Figure 4.5: The integral length scale $L_{\text {int }}$ vs. the Taylor scale Reynolds number $R e_{\lambda}$ for the background flow and the wake at $9.6 \mathrm{D}$ at tunnel speeds $U_{\infty}=1.3,4.9,8.3$, $10.6,12.0$, and $15.8 \mathrm{~m} \mathrm{~s}^{-1}$.

phase flow wake with sub-inertial tracer particles; and rows $\Phi_{1.2}, \Phi_{1.7}$, and $\Phi_{2}$ show contours of inertial particle behavior with water flow rates of $1.2,1.7$, and $2 \mathrm{~L} \mathrm{~min}^{-1}$ and corresponding particle volume fractions of $4.26 \times 10^{-6}, 6.03 \times 10^{-6}$, and $7.09 \times 10^{-6}$ respectively. The center of the disk is at $y / D=0$ and the flow is from the left. In Figure 4.9, pairs of columns show normalized values of mean streamwise velocity $\bar{u} / U_{\infty}$ and mean vertical velocity $\bar{v} / U_{\infty}$ for the near wake (left) and the far wake (right) of each column pair. Figure 4.10 has the same configuration as Figure 4.9, but pairs of

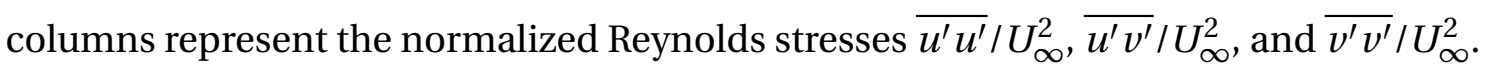
Vertical profiles of all quantities displayed in Figures 4.9 and 4.10 are represented in Figures 4.11 and 4.12, and are averaged spatially over $2 \mathrm{~mm}$. In Figures 4.11 and 4.12, 

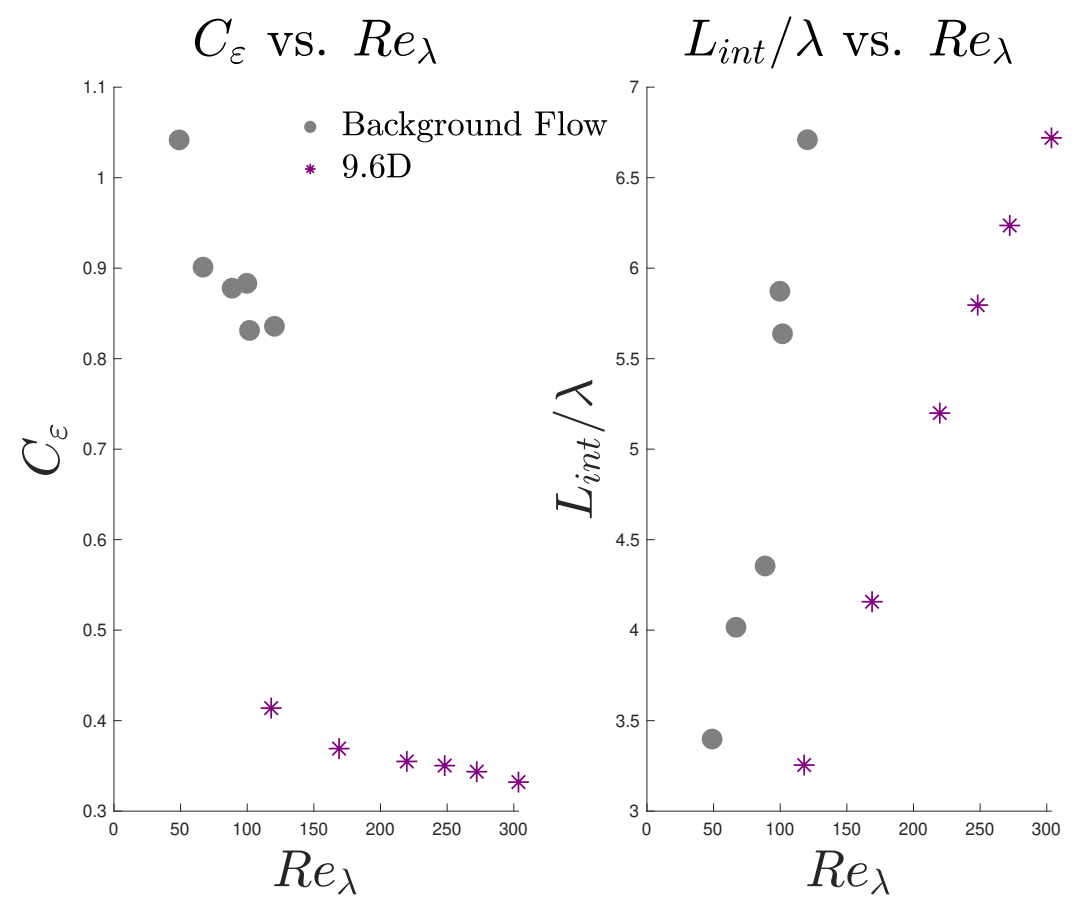

Figure 4.6: $C_{\varepsilon}$ and $L_{\text {int }} / \lambda$ are plotted vs. the Taylor scale Reynolds number $R e_{\lambda}$ for the background flow and the wake at $9.6 \mathrm{D}$ at tunnel speeds $U_{\infty}=1.3,4.9,8.3,10.6$, 12.0 , and $15.8 \mathrm{~ms}^{-1}$.

tracer particles (single-phase flow) are compared to three different volume fractions of inertial particles (two-phase flow) at 1D in the near wake (solid lines) and 9.6D in the far wake (dashed lines). Figure 4.11 compares the near and far wakes and Figure 4.12 zooms in on the far wake in order to compare different volume fractions.

In Figure 4.9, the near wake $\Phi_{0}$ velocity remains positive at the core of the wake, with a distinct steep velocity gradient around $\mathrm{y} / \mathrm{D} \sim 0.5$, above which is the free stream where $\bar{u} / U_{\infty}$ is unity. The lower half of the wake does not have a distinct edge due to the influence of the tube that attaches the disk to the tunnel floor. In contrast to the single-phase flow, the inertial particles reverse direction where they are trapped and recirculated behind the disk. Inertial particles shift the top of the wake to $y / D>0.7$. 


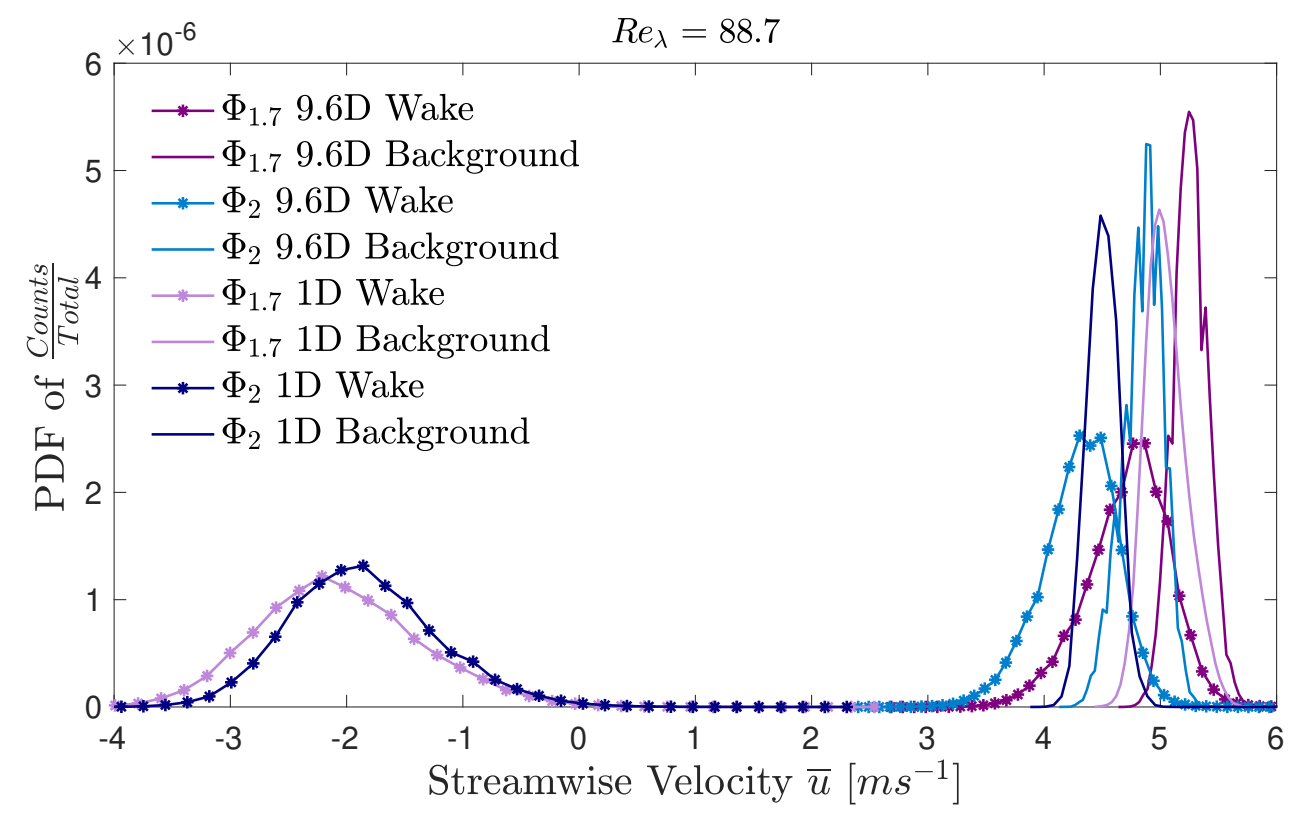

Figure 4.7: PDF of particle counts as a function of stream-wise velocity for one Reynolds number. Two volume fractions, two wake locations (1D and 9.6D) and wake vs. background flow are compared.

The two-phase flow near wakes grow wider, and increases particle negative velocity with increasing volume fraction. In the far wake single-phase flow $\left(\Phi_{0}\right)$, the core tapers more steeply and the wake shifts downward so that the center of the wake core is around $y / D \sim-0.3$. Again, the top edge of the wake is clearly defined and the bottom of the wake has moved lower than the region of interest, indicating the wake grows wider as it moves downstream. In the inertial particle cases, the far wake $\bar{u} / U_{\infty}$ is lower in magnitude and positive as particles move downstream. All three volume fractions have similar contours that show a reduction in $\bar{u} / U_{\infty}$ toward the bottom of the region of interest, suggesting the particle far wakes have shifted downward out of the region of interest. Figure 4.7 confirms that the wake is still present at $9.6 \mathrm{D}$, and the differences in particle velocity are small as one varies the volume fraction, which is in agreement 

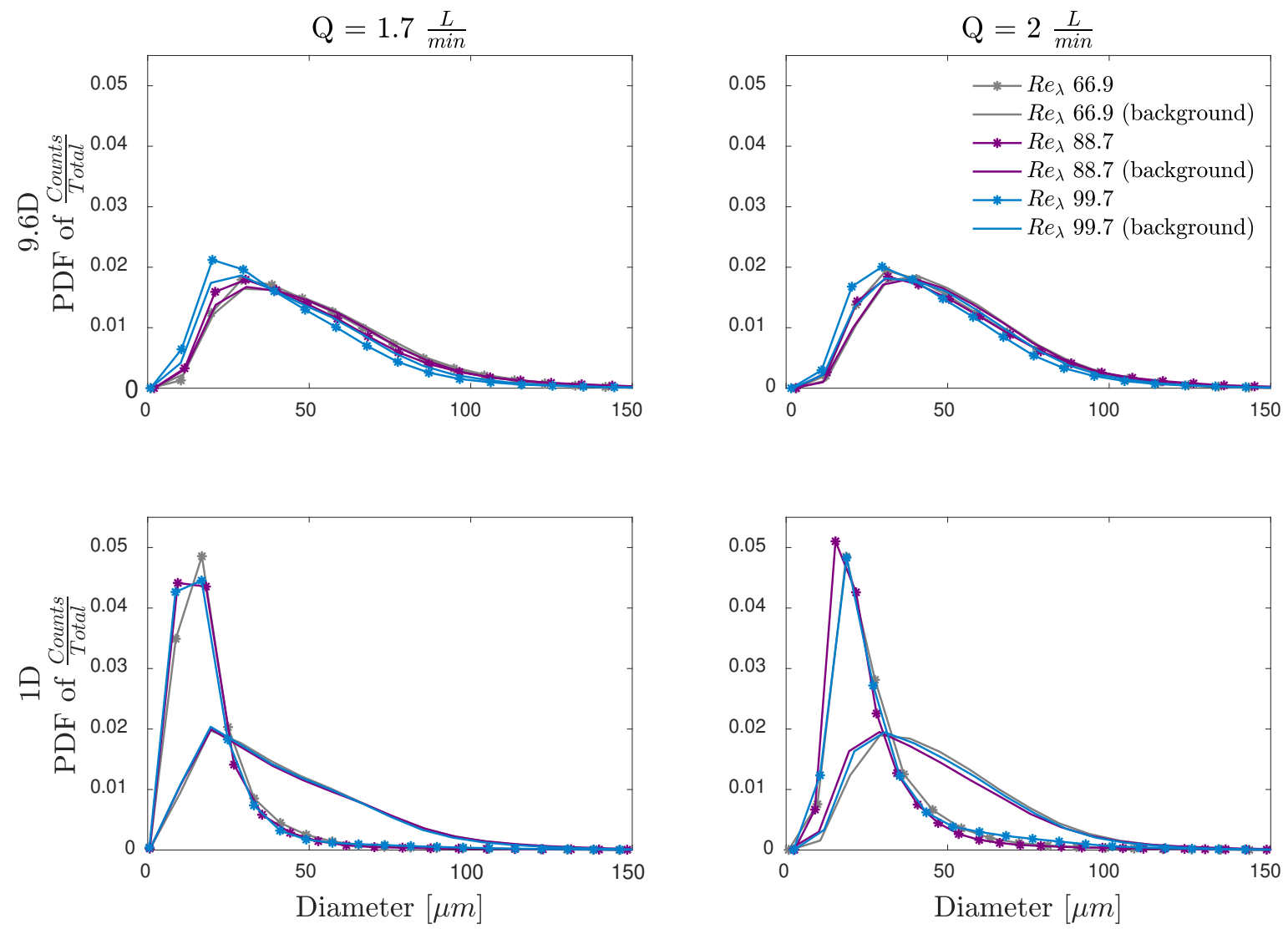

Figure 4.8: PDF of particle counts as a function of particle diameter for wakes at $1 \mathrm{D}$ and 9.6D (rows), and water flow rates of 1.7 and $2.0 \mathrm{Lmin}^{-1}$ (columns) for three different Reynolds numbers, and background flows (no wake).

with Figure 4.9. Particle $\bar{u} / U_{\infty}$ PIV measurements confirm particle recirculation in the near wake, and that these regions grow in size with increasing volume fraction.

As shown in the right column pair of Figure 4.9, the near wake normalized mean vertical velocity $\bar{v} / U_{\infty}$ remains zero above $\mathrm{y} / \mathrm{D} \sim 0.2$ in the single-phase flow case. Below $\mathrm{y} / \mathrm{D} \sim 0.2, \bar{v} / U_{\infty}$ is negative (downward), with the maximum downward flow at $\mathrm{y} / \mathrm{D} \sim-0.5$. Inertial particles reveal recirculation in the near wake, with a band of neg- 

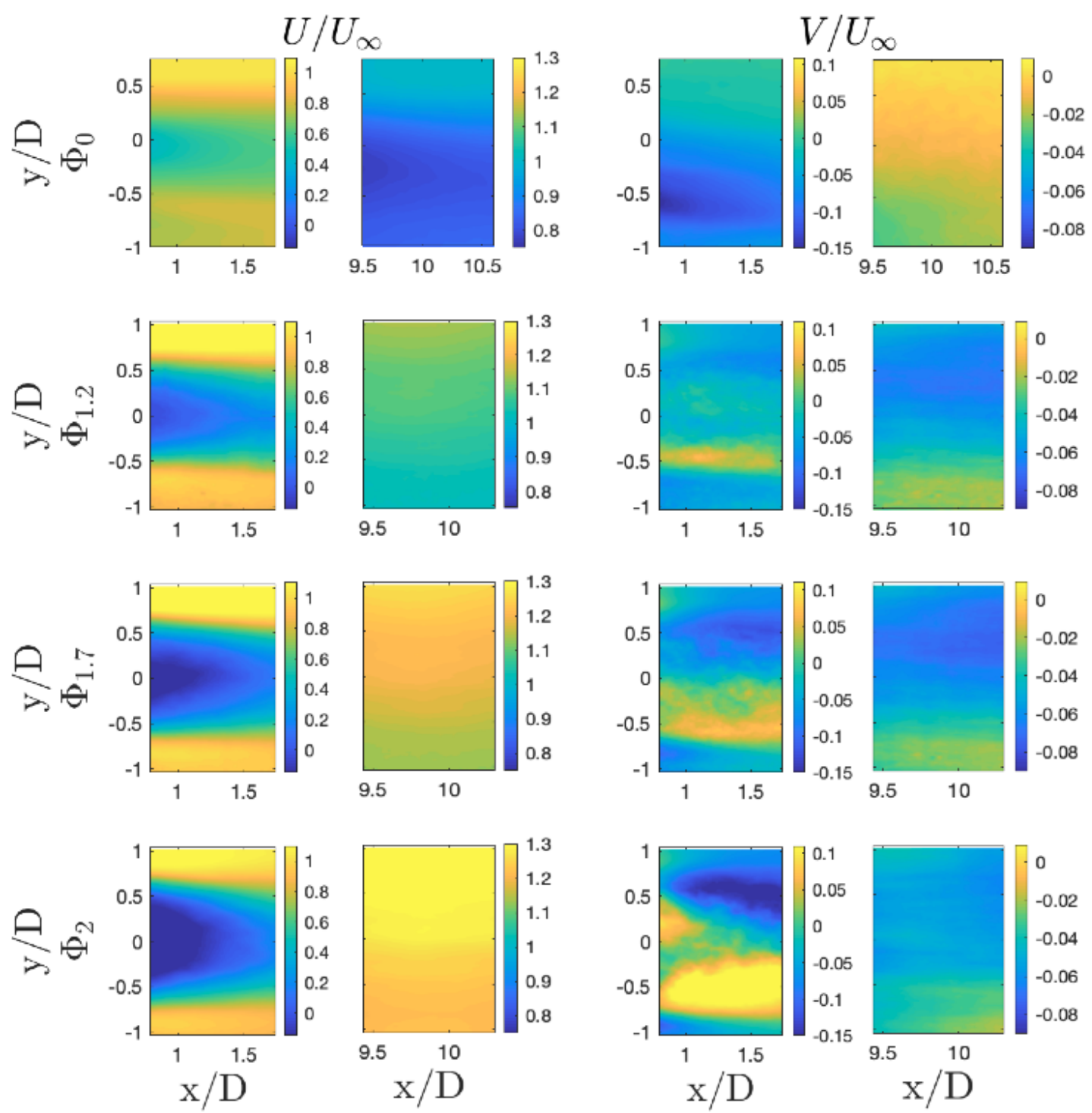

Figure 4.9: Normalized mean streamwise velocity $\bar{u} / U_{\infty}$ and mean vertical velocity $\bar{v} / U_{\infty}$ for single-phase flow and two-phase particle velocity fields with increasing volume fractions for $R e_{\lambda}=88.7$. The flow is from the left and column pairs represent the near and far wake regions of interest. Row $\Phi_{0}$ represents single-phase flow, while $\Phi_{1.2}, \Phi_{1.7}$, and $\Phi_{2}$ represent two-phase flow with water flow rates of 1.2, 1.7 , and $2 \mathrm{Lmin}^{-1}$ and volume fractions of $4.26 \times 10^{-6}, 6.03 \times 10^{-6}$, and $7.09 \times 10^{-6}$ respectively. Note that the near wake (left) and far wake (right) columns have different scales so that spatial features can be identified. 
ative $\bar{v} / U_{\infty}$ at the top of the wake at $\mathrm{y} / \mathrm{D} \sim 0.5$ and a band of positive $\bar{v} / U_{\infty}$ (upward) at the bottom of the wake at $y / D \sim-0.5$. These bands widen and intensify as the particle volume fraction is increased. This trend can also be observed in the second column of Figure 4.11, where the inertial particle 1D profiles have the opposite sign of the $\Phi_{0}$ single-phase flow grey line. In Figure 4.9 right column, the far wake $\bar{v} / U_{\infty}$ decreases with height for the $\Phi_{0}$ case, and a local minimum occurs below y/D $\sim-0.5$ and below $\mathrm{x} / \mathrm{D} \sim 10$. For the inertial particle cases, all three volume fractions exhibit similar magnitudes of negative $\bar{v} / U_{\infty}$ that approach but do not reach zero below $\mathrm{y} / \mathrm{D} \sim-0.5$. The absence of recirculation in the far wake allows for observation of the particle settling velocity. The second column in Figure 4.12 reveals the settling velocity is highest for the smallest volume fraction, and lowest for the greatest volume fraction. All far wake cases below y/D - 0.6 have similar $\bar{v} / U_{\infty}$ magnitude. The $\Phi_{1.2}$ and $\Phi_{1.7}$ cases have nearly identical profiles and magnitudes, while the greatest volume fraction $\left(\Phi_{2}\right)$ profile diverges from this pattern and has the lowest negative velocity. This shows dependence of settling velocity on volume fraction.

The left column pair of Figure 4.10 displays the normal Reynolds stress $\overline{u^{\prime} u^{\prime}} / U_{\infty}^{2}$, which represents the contributions of streamwise turbulence fluctuations to momentum within the single-phase flow wake. In the near wake, the single-phase flow normal stress is near zero at $\mathrm{y} / \mathrm{D} \sim 0.3$, and increases downward and upstream (left). The particle velocity fields show bands of normal stress at the top and bottom edges of the wake, at $\mathrm{y} / \mathrm{D} \sim \pm 0.5$. Interestingly, the middle volume fraction $\Phi_{1.7}$ differs from the other particle cases, with greater stress in the lower band of the wake, and less stress in the upper band. The behaviour of $\overline{u^{\prime} u^{\prime}} / U_{\infty}^{2}$ in the far wake single-phase flow is different than the near wake, where a band of higher $\overline{u^{\prime} u^{\prime}} / U_{\infty}^{2}$ is clearly visible at $\mathrm{y} / \mathrm{D} \sim 0.25$, 
and it increases as it moves downstream. Note that the vertical line of slightly higher values at $\mathrm{x} / \mathrm{D} \sim 10.2$ is due to a laser reflection. Generally, the far wake inertial particle cases have lower magnitudes for the middle volume fraction $\Phi_{1.7}$, and show larger bands of greater normal stress in the lower part of the region of interest below $y / D$ $\sim-0.5$. All inertial particle cases have a thinner but distinct band of higher $\overline{u^{\prime} u^{\prime}} / U_{\infty}^{2}$ at $y / D \sim 0.5$. Inertial particle velocity fields have different structures than the singlephase flow, where particles tend to have greater $\overline{u^{\prime} u^{\prime}} / U_{\infty}^{2}$ at the top and bottom edges of the wake.

The middle column pair of Figure 4.10 shows shearing Reynolds stress $\overline{u^{\prime} v^{\prime}} / U_{\infty}^{2}$, which represents vertical fluctuation influence on streamwise turbulent momentum for single-phase flows. In the near wake single-phase flow, the shearing stress is negative at $\mathrm{y} / \mathrm{D} \sim 0.1$ and $\sim 0.5$, and three bands of positive shear stress appear at $\mathrm{y} / \mathrm{D}$ $\sim-0.2,-0.5$, and -0.9 . The inertial particle velocity fields show pronounced bands of low and high $\overline{u^{\prime} v^{\prime}} / U_{\infty}^{2}$ with a negative band at $\mathrm{y} / \mathrm{D} \sim 0.5$ and a wider positive band at y/D - 0.5. As with $\overline{u^{\prime} u^{\prime}} / U_{\infty}^{2}$, the upper band of positive $\overline{u^{\prime} v^{\prime}} / U_{\infty}^{2}$ is thinnest in the near wake $\Phi_{1.7}$ case. In the single-phase flow far wake, bands of positive and negative shear stress are wider with less defined edges as the wake expands downstream. The $\Phi_{0}$ case has an upper band of negative shear stress centered at $\mathrm{y} / \mathrm{D} \sim 0.2$ and a lower band of positive shear stress centered at $\mathrm{y} / \mathrm{D} \sim-0.7$, with bands of zero shear stress at $\mathrm{y} / \mathrm{D}>0.5$ and $\sim-0.25$. The far wake particle velocity fields also have bands of negative shear stress at the top of the wake, and positive shear stress below, but these bands have moved away from the center of the wake, with the upper band centered around $\mathrm{y} / \mathrm{D} \sim 0.53$ and less defined lower bands of positive shear stress starting below $\mathrm{y} / \mathrm{D}$ $\sim-0.45$. Both the $\Phi_{1.2}$ and $\Phi_{1.7}$ cases have a band of zero shear stress at $\mathrm{y} / \mathrm{D} \sim 0$, but 

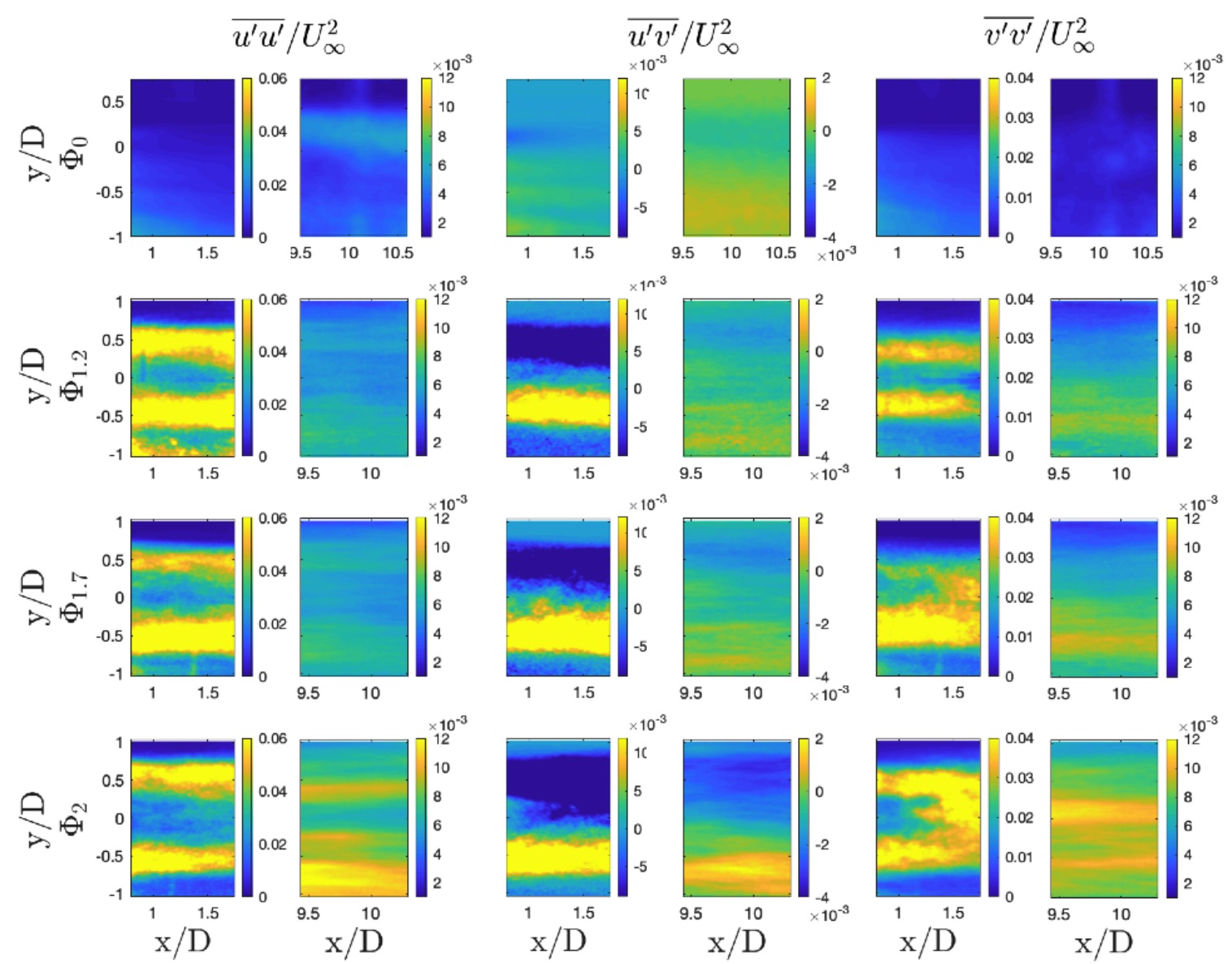

Figure 4.10: Normalized mean Reynolds stresses $\overline{u^{\prime} u^{\prime}} / U_{\infty}^{2}, \overline{u^{\prime} v^{\prime}} / U_{\infty}^{2}$, and $\overline{v^{\prime} v^{\prime}} / U_{\infty}^{2}$ for single-phase flow and two-phase particle velocity fields with increasing volume fractions for $R e_{\lambda}=88.7$. The flow is from the left and column pairs represent the near and far wake regions of interest. Row $\Phi_{0}$ represents single-phase flow, while $\Phi_{1.2}, \Phi_{1.7}$, and $\Phi_{2}$ represent two-phase flow with water flow rates of $1.2,1.7$, and 2 $\mathrm{Lmin}^{-1}$ and volume fractions of $4.26 \times 10^{-6}, 6.03 \times 10^{-6}$, and $7.09 \times 10^{-6}$ respectively. Note that the near wake (left) and far wake (right) columns have different scales so that spatial features can be identified. 
this band moves downward to $\mathrm{y} / \mathrm{D} \sim-0.3$ for the $\Phi_{2}$ case. The Reynolds shear stress is clearly shown in Figure 4.11 for the near wake, where particles cases have distinct peaks near $y / D \sim \pm 0.5$. While Figure 4.12 shows the far wake particle Reynolds shear stress is greater in magnitude and has a different profile shape for the $\Phi_{2}$ case. The particle velocity fields show different patterns of $\overline{u^{\prime} v^{\prime}} / U_{\infty}^{2}$ than the single-phase flow, with bands of negative and positive particle field shear stress concentrating at the top and bottom of the wake, respectively.

The third column pair in Figure 4.10 shows $\overline{v^{\prime} v^{\prime}} / U_{\infty}^{2}$, which is the vertical Reynolds normal stress and represents energy contributions within the single-phase flow wake from the vertical turbulent fluctuations. The single-phase flow $\overline{v^{\prime} \nu^{\prime}} / U_{\infty}^{2}$ in the near wake, is about zero above $\mathrm{y} / \mathrm{D} \sim 0.25$, and increases as you move downward and to the left. In the far wake, the magnitude of the single-phase $\overline{v^{\prime} v^{\prime}} / U_{\infty}^{2}$ is an order of magnitude lower than the near wake and has expanded slightly upward. Note that in the far wake region of interest, the vertical line of slightly higher values at $\mathrm{x} / \mathrm{D} \sim 10.2$ is due to a laser reflection. The near wake particle velocity field $\overline{v^{\prime} v^{\prime}} / U_{\infty}^{2}$ forms bands at the top and bottom of the wake, but less distinct than $\overline{u^{\prime} v^{\prime}} / U_{\infty}^{2}$, and increases with increasing volume fraction. In the near wake $\Phi_{1.2}$ case, two distinct bands of higher $\overline{v^{\prime} v^{\prime}} / U_{\infty}^{2}$ are centered behind the disc at $\mathrm{y} / \mathrm{D} \sim 0.3$ and -0.3 . In the far wake, the greatest increase in $\overline{v^{\prime} v^{\prime}} / U_{\infty}^{2}$ occurs in the lowest volume fraction with the band of greatest intensity around $\mathrm{y} / \mathrm{D} \sim-0.6$. This band is reduced in intensity in the $\Phi_{1.7}$ case and then splits into two distinct bands in the $\Phi_{2}$ case with the greatest intensity shifting upward to $\mathrm{y} / \mathrm{D} \sim 0$ and a thinner band remaining at $\mathrm{y} / \mathrm{D} \sim-0.6$. Figure 4.12 shows that although the changes in far wake particle magnitudes are small, the $\Phi_{2}$ case has greater $\overline{v^{\prime} v^{\prime}} / U_{\infty}^{2}$ in the top half of the wake, and lower $\overline{v^{\prime} v^{\prime}} / U_{\infty}^{2}$ below. Figure $4.12 \bar{v} / U_{\infty}$ and 

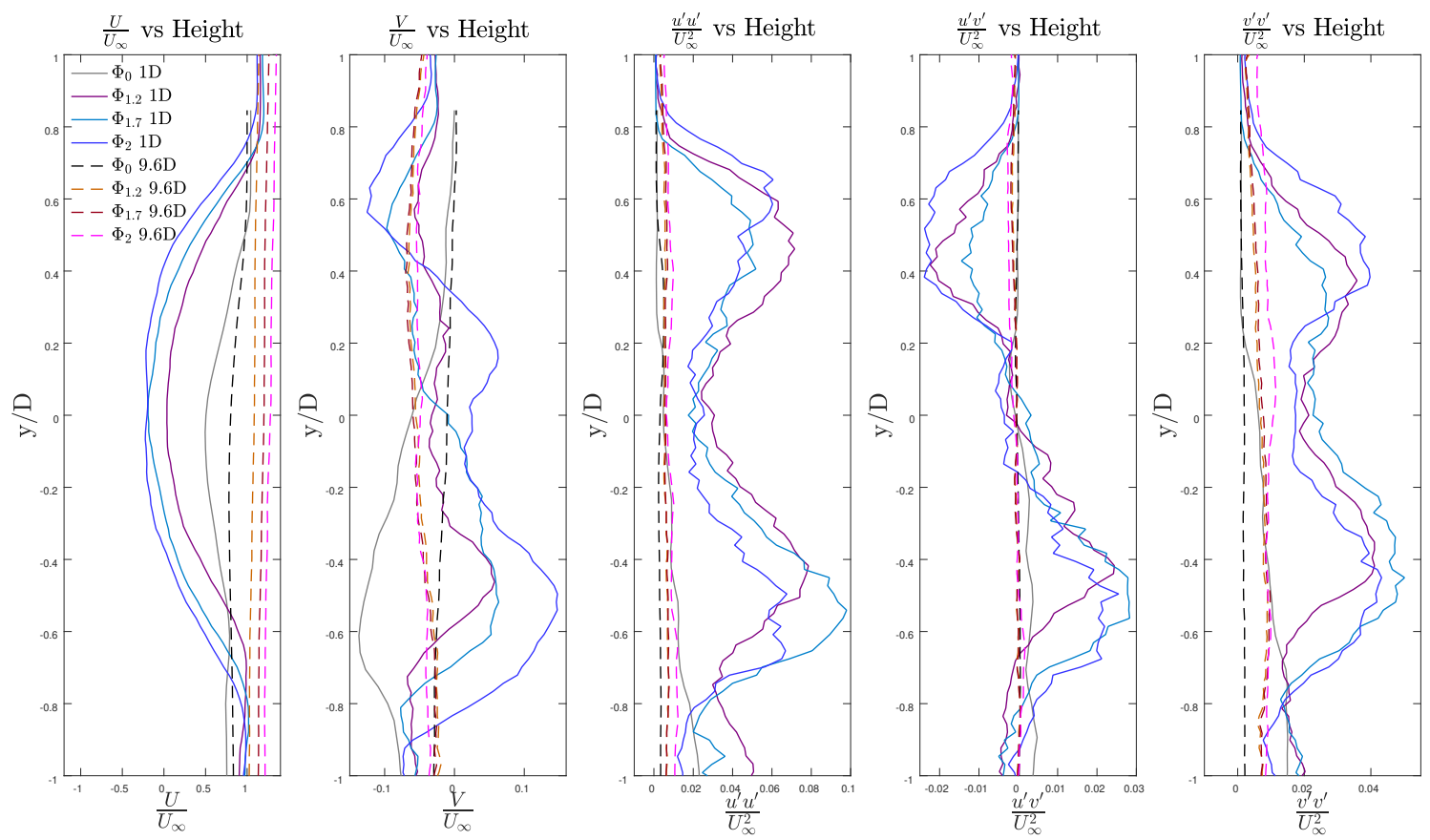

Figure 4.11: Normalized mean turbulence statistics as a function of height at $1 \mathrm{D}$ (solid lines) and 9.6D (dashed lines). $\Phi_{0}$ represents single-phase flow, while $\Phi_{1.2}$, $\Phi_{1.7}$, and $\Phi_{2}$ represent two-phase flow with water flow rates of $1.2,1.7$, and 2 $\mathrm{Lmin}^{-1}$ and volume fractions of $4.26 \times 10^{-6}, 6.03 \times 10^{-6}$, and $7.09 \times 10^{-6}$ respectively. Flow direction is from the left, and $y / D=0$ is the center of the disk.

$\overline{v^{\prime} v^{\prime}} / U_{\infty}^{2}$ profiles show that the far wake inertial particle cases are similar in the lower part of the wake, but the $\Phi_{2}$ case diverges from the other two with a lower settling velocity and higher $\overline{v^{\prime} v^{\prime}} / U_{\infty}^{2}$.

Comparison of turbulence statistics between single-phase and two-phase flow in Figures 4.9 - 4.12 gives insight into how the turbulent wake and particles interact. There are distinct differences between the single-phase flow and the inertial particle velocity fields, as well as differences in the particle fields with changing volume fraction. 

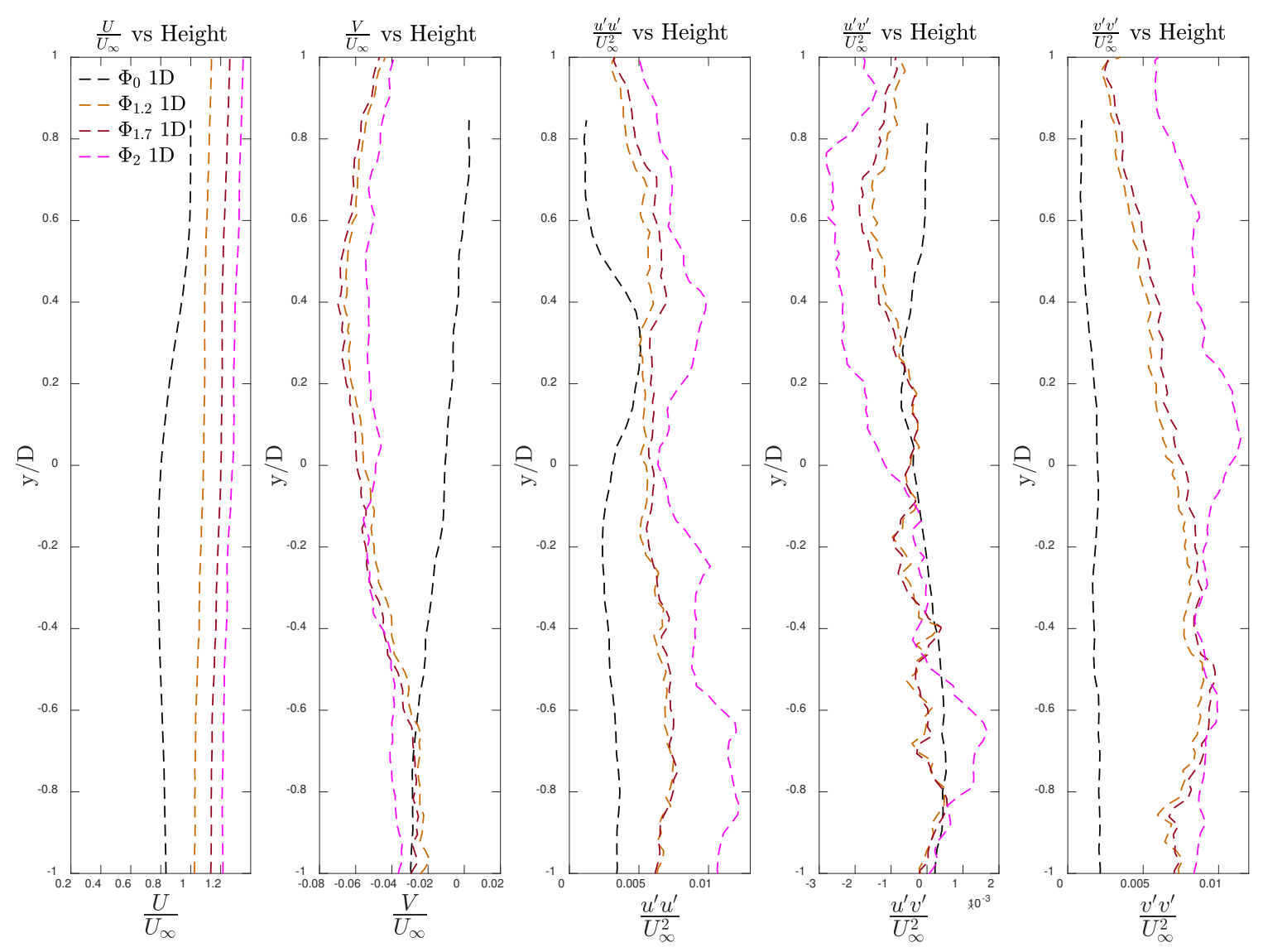

Figure 4.12: Zoomed in normalized mean turbulence statistics as a function of height at 9.6D. $\Phi_{0}$ represents single-phase flow, while $\Phi_{1.2}, \Phi_{1.7}$, and $\Phi_{2}$ represent two-phase flow with water flow rates of $1.2,1.7$, and $2 \mathrm{~L} \mathrm{~min}^{-1}$ and volume fractions of $4.26 \times 10^{-6}, 6.03 \times 10^{-6}$, and $7.09 \times 10^{-6}$ respectively. Flow direction is from the left, and $\mathrm{y} / \mathrm{D}=0$ is the center of the disk. 


\section{Chapter 5}

\section{Discussion}

The background flow turbulence and the wake without inertial particles were characterized with hot-wire anemometry measurements (Figures 3.5 - 4.6). Hot-wire results confirm HIT in the far wake and background flow (no wake), with anisotropic behaviour in the near wake. $\lambda$ and $\eta$ were measured to be smaller in the wake, and decrease with increasing $R e_{\lambda}$. As expected, the single-phase wake reduces $\bar{u}$, increases turbulence intensity, and generates more turbulent dissipation than the background flow.

Polydisperse inertial particles were introduced to the flow in particle volume frac-

tions from $\Phi_{V}=2.95 \times 10^{-6}-1.22 \times 10^{-5}$. PDI measurements (Figures $4.7-4.8$ ) reveal the wake reduces particle horizontal velocities by $140 \%$ in near wake and $7-12 \%$ in far wake. Particles have negative horizontal velocity at 1D, indicating recirculation in the near wake, and reduced particle velocities compared to the background flow show the wake is still present at 9.6D downstream. The PDI measurements also show that increasing $\Phi_{\nu}$ increases the probability of larger particle diameters. Background flow particle diameters were measured on average to be $9 \mu \mathrm{m}$ bigger in the larger $\Phi_{V}$ case, and may be due to droplet coalescence. Particles are smaller in the wake than the background flow, and smallest in the near wake. This indicates the near wake recircu- 
lation tends to trap smaller particles. Interestingly, the near wake particle diameters are smallest at $R e_{\lambda}=88.7$, and larger at $R e_{\lambda}=66.9$ and 102, suggesting a Reynolds number influence on particle diameter entrainment.

Both PIV measurements and PDI measurements (Figures 4.9 and 4.7) show particle $\bar{u} / U_{\infty}$ is reversed in the near wake. PIV measurements reveal that the region of negative particle $\bar{u} / U_{\infty}$ increases in size in the y/D direction with increasing $\Phi_{V}$. Particle recirculation in the near wake is also apparent in the $\bar{v} / U_{\infty}$ contour plots, where particles in the top half of the wake have negative vertical velocity (downward), and particles in the lower half of the wake have positive vertical velocity (upward). These regions of particle $\bar{v} / U_{\infty}$ widen and intensify with increasing volume fraction.

In the far wake, particle vertical velocity (Figures 4.9 and 4.12 ) decreases with increasing volume fraction, which is in agreement with Sumbekova et al. and Good et al. [24] [9]. This result is interesting, and occurs in the top half of the wake where $\overline{u^{\prime} v^{\prime}} / U_{\infty}^{2}$ is negative, and $\overline{u^{\prime} u^{\prime}} / U_{\infty}^{2}$ and $\overline{v^{\prime} v^{\prime}} / U_{\infty}^{2}$ are greatest for the greatest volume fraction. Possible explanations for this behaviour are nonlinear drag on particles, the loitering effect where falling particles spend more time in regions with upward flow, or regions with low preferential sweeping. [21] Preferential sweeping is enhancement of settling velocity where inertial particles accumulate on the downward side of eddies. Rosa et al. found in DNS simulations that when preferential sweeping is disabled, the settling velocity of droplets is reduced, and droplets smaller than $55 \mu \mathrm{m}$ are sensitive to $\varepsilon$. [21]

In the single-phase flow wake in Figure 4.10, $\overline{u^{\prime} u^{\prime}} / U_{\infty}^{2}, \overline{u^{\prime} v^{\prime}} / U_{\infty}^{2}$, and $\overline{v^{\prime} v^{\prime}} / U_{\infty}^{2}$ are greater in magnitude in the near wake and smaller in the far wake, indicating streamwise fluctuations and Reynolds shear stress decrease as the wake moves downstream. 
Note the contour scales are different for the near and far wake plots in Figure 4.10. The particle $\overline{u^{\prime} u^{\prime}} / U_{\infty}^{2}, \overline{u^{\prime} v^{\prime}} / U_{\infty}^{2}$, and $\overline{v^{\prime} v^{\prime}} / U_{\infty}^{2}$ are also greater in the near wake and concentrated where $\bar{u} / U_{\infty}$ has a steep gradient near $\mathrm{y} / \mathrm{D} \sim \pm 0.5$. Regions of stress at the top and bottom edges of the single-phase flow wake are where the greatest particle field stresses are located. Figure 4.12 shows the far wake where $\overline{u^{\prime} u^{\prime}} / U_{\infty}^{2}$ is greatest for the greatest volume fraction. Here, particle $\overline{u^{\prime} v^{\prime}} / U_{\infty}^{2}$ and $\overline{v^{\prime} v^{\prime}} / U_{\infty}^{2}$ are similar in magnitude for all volume fractions in the lower part of the wake, and deviate for the greatest volume fraction in the top half of the wake, suggesting an influence on the settling velocity.

The lack of symmetry between values at $\mathrm{y} / \mathrm{D} \sim \pm 0.5$ may be due to the presence of the tube that attaches the disk to the tunnel floor and the fact that particles are falling as they move downstream. Although the particle diameters are smaller than $\eta$ for all PIV results presented, the Stokes numbers increase with increasing $\varepsilon$, and $\varepsilon$ increases in the presence of the wake. The calculated near and far wake Stokes numbers are 0.77 and 1.7, respectively compared to the background flow (no wake) of 0.58 . This suggests that the Stokes number influences the particle/turbulence interaction, and particles could be affecting the turbulence in the wake. Previous studies have proposed that heavy particles in turbulence tend to be centrifuged from the center of vortices and trapped in convergent regions of the flow with high strain and low vorticity. [16] This could lead to concentrated regions of higher $\Phi_{V}$ that may affect the flow (two-way coupling) and explain why the particle fields have distinctly different behaviour than the background flow. 


\section{Chapter 6}

\section{Conclusion}

This study presents the findings of a wind tunnel experiment investigating the behaviour of inertial particles in the turbulent wake of a porous disk. The incoming flow was characterized as homogeneous, isotropic turbulence, and the wake of the disk was measured in regions around $1 \mathrm{D}$ and 9.6D downstream. Poly-dispersed particles (water droplets) were injected uniformly upstream of the disk at varying volume

fractions. Both single-phase and two-phase flow measurements were taken with hot wire anemometry, phase Doppler interferometry (PDI), and particle image velocimetry (PIV) to investigate the wake particle interaction.

Hot-wire measurements showed the local mean stream-wise velocity is reduced by $54 \%-66 \%$ at $1 \mathrm{D}$ and $7 \%-19 \%$ at $9.6 \mathrm{D}$. The wake increases turbulence intensity by $869 \%-987 \%$ at 1D and $205 \%-246 \%$ at $9.6 D$. In single-phase flow, the wake produces smaller turbulent eddies and increases energy dissipation. Essentially, the disk acts as a turbulence generator, with anisotropic flow in the near wake.

Analysis of PDI measurements found the most probable particle diameters range from 13-41 $\mu \mathrm{m}$, and that particle diameters vary with particle volume fraction and location within the wake. At $1 \mathrm{D}$ the most probable particle diameter was $17 \mu \mathrm{m}$, while the average particle diameter in the far wake was $30 \mu \mathrm{m}$. Particle velocities were re- 
versed at $1 \mathrm{D}$ and reduced by $141 \%$ - $144 \%$. At $9.6 \mathrm{D}$ particle velocities were reduced by $7.4 \%-12.2 \%$ compared to particles in the background two-phase flow (no wake). Recirculation and trapping of smaller particles occurs at 1D while the wake still influences particles at 9.6D downstream.

PIV contours also confirm a reversal of particle $\bar{u} / U_{\infty}$ at $1 \mathrm{D}$, and a reduced stream wise velocity at $9.6 \mathrm{D}$. Particle field $\bar{v} / U_{\infty}$ shows recirculation at $1 \mathrm{D}$ with horizontal bands of downward velocity in the top half of the wake and upward velocity in the lower half. Particle Reynolds stress magnitudes tend to be greatest at $\mathrm{y} / \mathrm{D} \sim \pm 0.5$ at the 'edges' of the wake where the gradient of $\bar{u} / U_{\infty}$ is steep and shearing occurs. This supports the idea that particles cluster in regions of high strain and low vorticity.

PIV vertical profiles reveal that particle quantity magnitudes are greater in the near wake than particle magnitudes in the far wake, a region of higher turbulence intensity.

It is also shown that at 9.6D, particle downward velocity was found to decrease with increasing volume fraction in the upper portion of the wake. This occurs where shear stress is negative and vertical stress increases with increasing volume fraction.

These results show that at $R e_{D}=6.50 \times 10^{4}$, there are distinct differences between turbulence statistics of a single-phase wake behind a porous disk, and the behaviour of inertial particles at volume fractions of $\Phi_{V}=2.95 \times 10^{-6}-1.22 \times 10^{-5}$ when added to the wake. Additionally, there are different structures in the near and far wake regions. Whether particle settling velocity is enhanced or reduced is greatly debated in the literature, and depends on many parameters such as drag nonlinearity, vortex trapping/centrifuging, loitering, preferential sweeping, particle size, Stokes number, $\operatorname{Re}_{\lambda}$, dissipation rate $\varepsilon$, and volume fraction. These parameters vary throughout the wake and are a starting point for further exploration. 


\section{Chapter 7}

\section{Future Work}

Future work should continue the investigation of dependence on $R e_{\lambda}$, as PIV data was taken for two other Reynolds numbers and is not presented here.

Quadrant analysis, a method that determines whether two different fluctuating variables ( $u^{\prime}$ and $v^{\prime}$ in this case) are correlated by plotting their joint frequency of occurrence will be performed to explore shear effects. The Reynolds stress term, $u^{\prime} v^{\prime}$ can be described in four events: outward interactions Q1 ( $u>0$ and v $>0$ ), ejections Q2 ( $u$ $<0$ and $\mathrm{v}>0)$, inward interactions Q3 $(\mathrm{u}<0$ and $\mathrm{v}<0)$, and sweeps Q4 $(\mathrm{u}>0$ and $\mathrm{v}$ $<0$ ). These can be physically interpreted as instantaneous directionality compared to the mean flow.

Voronoi analysis, a statistical and qualitative way observing particle clustering, should also be implemented. In 2D space, polygonal cells are created enclosing defined points. Voronoi distributions are compared to Poisson distributions to identify preferential concentration of particles. The size of the cells are dependent on proximity of individual particles. A collection of smaller adjacent cells represents locally high concentrations (clusters) of particles, while large cells represent areas with larger distance between particles and represent low concentrations (voids).

The settling velocity and dependence on Stokes number should also be explored, 
as particle diameters, and $\varepsilon$ were found to vary by location within the wake. The particle relative velocities and particle Reynolds numbers should be calculated. The goal would be to determine if clusters occur in areas of high shear stress, and if clustering of particles could lead to Stokes numbers and volume fractions capable of modifying turbulence (two-way coupling) and give more insight into the differences observed between the single-phase flow and inertial particle velocity fields within the wake. 


\section{Bibliography}

[1] A. Aliseda, A. Cartellier, F. Hainaux, And J. C. Lasheras, Effect of preferential concentration on the settling velocity of heavy particles in homogeneous isotropic turbulence, Journal of Fluid Mechanics, 468 (2002), pp. 77-105.

[2] F. Aloui, M. Kardous, R. Cheker, and S. Ben Nasrallah, Study of the wake induced by a porous disc, 21eme Congres Francais de Mecanique, (2013).

[3] S. Aubrun, S. LOYER, P. HANCOCK, And P. HAYden, Wind turbine wake properties: Comparison between a non-rotatingsimplified wind turbine model and a rotating model, Journal of Wind Engineeringand Industrial Aerodynamics, (2013).

[4] S. Balachandar And J. K. Eaton, Turbulent Dispersed Multiphase Flow, Annual Review of Fluid Mechanics, 42 (2010), pp. 111-133.

[5] M. Bourgoin And H. XU, Focus on dynamics of particles in turbulence, New Journal of Physics, 16 (2014), p. 085010.

[6] E. H. CAMP AND R. B. CAL, Mean kinetic energy transport and event classification in a model wind turbine array versus an array of porous disks: Energy budget and octant analysis, PHYSICAL REVIEW FLUIDS 1, 044404 (2016), (2016).

[7] T. Dairay, M. Obligado, And J. C. Vassilicos, Nonequilibrium scalings of turbulent wakes, PHYSICAL REVIEW FLUIDS 1, 044409 (2016), (2016).

[8] W. K. GeORGE, The Self-Preservation of Turbulent Flows and Its Relation to Initial Conditions and Coherent Structures, 1989.

[9] G. H. Good, P. J. Ireland, G. P. Bewley, E. Bodenschatz, L. R. Collins, and Z. WARHAFT, Settling regimes of inertial particles inisotropic turbulence, J. Fluid Mech., vol.759, R3,doi:10.1017/jfm.2014.602 (2014).

[10] W. HUMPHRIES AND J. H. VINCENT, Experiments to investigate transport processes in the near wakes of disks in turbulent air flow, Journal of Fluid Mechanics, (1976). 
[11] N. H. C. HWANG AND L. V. BALDWIn, Decay of Turbulence in Axisymmetric Wakes, Journal of Basic Engineering, (1969).

[12] D. E. JACOBER AND M. J. MATTESON, The Collection of Aerosols from Gas Streams by Impaction on Multiple Spherical Targets, Aerosol Science and Technology, 4 (1985), pp. 433-443.

[13] P. B. Johansson And W. K. George, Proper orthogonal decomposition of anaxisymmetric turbulent wake behind a disk, PHYSICS OF FLUIDS, 17 (2002).

[14] P. B. V. Johansson And W. K. GeORGE, The far downstream evolution of the highReynolds-number axisymmetric wake behind a disk. Part 1. Single-point statistics, Journal of Fluid Mechanics, 555 (2006), p. 363.

[15] L. E. M. Lignarolo, D. Ragni, C. J. Ferreira, And G. J. W. van Bussel, Experimental comparison of a wind-turbine and of an actuator-disc near wake, Journal of Renewable and Sustainable Energy, (2016).

[16] R. Monchaux, M. Bourgoin, and A. Cartellier, Analyzing preferential concentration and clustering of inertial particles in turbulence, International Journal of Multiphase Flow, (2011).

[17] S. NADERI AND F. TORABI, Numerical investigation of wake behind a HAWT using modifiedactuator disc method, Energy Conversion and Management, (2017).

[18] J. Nedić, J. C. Vassilicos, and B. Ganapathisubramani, Axisymmetric Turbulent Wakes with New Nonequilibrium Similarity Scalings, PHYSICAL REVIEW LETTERS, (2013).

[19] M. Obligado, A. Cartellier, A. Aliseda, T. Calmant, and N. De Palma, Study on preferential concentration of inertial particles in homogeneous isotropic turbulence via Big-Data techniques, PHYSICAL REVIEW FLUIDS 2, 024302 (2017), (2019).

[20] B. W. Roberts, Drag and Pressure Distribution on a Family of Porous, Slotted Disks, J. Aircraft, 17 (1980).

[21] B. Rosa, H. Parishani, O. Ayala, AND L.-P. WANG, Settling velocity of small inertial particles in homogeneous isotropic turbulence from high-resolution DNS, International Journal of Multiphase Flow, 83 (2016), pp. 217-231.

[22] S. E. Smith, K. N. Travis, H. Djeridi, M. Obligado, and R. B. Cal, Dynamic effects of inertial particles on the wake recovery of a model wind turbine, Renewable Energy, 164 (2021), pp. 346-361. 
[23] D. Soto, H.-L. Girard, A. Le Helloco, T. Binder, D. Quéré, and K. K. VARANASI, Droplet fragmentation using a mesh, Physical Review Fluids, 3 (2018), p. 083602. Publisher: American Physical Society.

[24] S. Sumbekova, A. Aliseda, A. Cartellier, And M. Bourgoin, Clustering and Settling of Intertial Particles in Turbulence, Proceedings of the 5th International Conference on Jets, Wakes and Separated Flows (ICJWSF2015), (2016).

[25] W. Thielicke And E. Stamhuis, PIVlab - Towards User-friendly, Affordable and Accurate Digital Particle Image Velocimetry in MATLAB, Journal of Open Research Software, 2 (2014), p. e30. Number: 1 Publisher: Ubiquity Press.

[26] F. Toschi And E. Bodenschatz, Lagrangian Properties of Particles in Turbulence, Annual Review of Fluid Mechanics, 41 (2009), pp. 375-404.

[27] A. TownsEnd, The structure of turbulent shear flow, 1976.

[28] J. C. Vassilicos, Dissipation in Turbulent Flows, Annual Review of Fluid Mechanics, 47 (2015), pp. 95-114. _eprint: https://doi.org/10.1146/annurev-fluid010814-014637. 